TRANSACTIONS OF THE

AMERICAN MATHEMATICAL SOCIETY

Volume 349, Number 12, December 1997, Pages 4857-4895

S 0002-9947(97)01814-X

\title{
FROBENIUS EXTENSIONS OF SUBALGEBRAS OF HOPF ALGEBRAS
}

\author{
D. FISCHMAN, S. MONTGOMERY, AND H.-J. SCHNEIDER
}

\begin{abstract}
We consider when extensions $S \subset R$ of subalgebras of a Hopf algebra are $\beta$-Frobenius, that is Frobenius of the second kind. Given a Hopf algebra $H$, we show that when $S \subset R$ are Hopf algebras in the Yetter-Drinfeld category for $H$, the extension is $\beta$-Frobenius provided $R$ is finite over $S$ and the extension of biproducts $S \star H \subset R \star H$ is cleft.

More generally we give conditions for an extension to be $\beta$-Frobenius; in particular we study extensions of integral type, and consider when the Frobenius property is inherited by the subalgebras of coinvariants.

We apply our results to extensions of enveloping algebras of Lie coloralgebras, thus extending a result of Bell and Farnsteiner for Lie superalgebras.
\end{abstract}

\section{INTRODUCTION}

In this paper we consider when various extensions $S \subset R$ of subalgebras of a Hopf algebra are $\beta$-Frobenius; such extensions generalize the usual notion of Frobenius extensions by having the module action on one side twisted by an automorphism $\beta$ of $S$. It was already known that any extension of finite-dimensional Hopf algebras is $\beta$-Frobenius (a result of the third author [Sch 92]) as is any finite extension $U(K) \subset U(L)$ of enveloping algebras of Lie superalgebras (a result of Bell and Farnsteiner $[\mathrm{BF}])$. Note that $U(L)$ is not an ordinary Hopf algebra, but rather a Hopf algebra in the category of $\mathbb{Z}_{2}$-graded modules. These results were an important motivation for this paper, and raised the question as to when an extension of Hopf algebras in a category was $\beta$-Frobenius.

One of the main results of this paper is that an extension $S \subset R$ of Hopf algebras of finite index in the Yetter-Drinfeld category ${ }_{H}^{H} \mathcal{Y D}$ for a given Hopf algebra $H$ is $\beta$-Frobenius provided that the associated extension of Hopf algebras $S \star H \subset R \star H$ (the biproducts of $S$ and $R$ with $H$ ) has a normal basis (Theorem 5.6); this will happen whenever $R$ and $H$ are finite-dimensional, or when $R \star H$ is pointed. As an application we are able to generalize the $[\mathrm{BF}]$ result to Lie coloralgebras: if $U(K) \subset U(L)$ is a finite extension of enveloping algebras of Lie coloralgebras, then it is $\beta$-Frobenius. Moreover we give an explicit description of the automorphism $\beta$ of $U(K)$, and of the Frobenius homomorphism $f: U(L) \rightarrow U(K)$ (Corollary 6.3).

Along the way we prove a number of other results about $\beta$-Frobenius extensions and conditions that ensure that an extension is $\beta$-Frobenius.

In Section 1 we give a short direct proof of the fact that an extension $B \subset A$ of finite-dimensional Hopf algebras is always $\beta$-Frobenius; moreover we give explicit

Received by the editors December 10, 1995.

1991 Mathematics Subject Classification. Primary 16W30; Secondary 17B35, 17 B37.

(C)1997 American Mathematical Society 
formulae for the automorphism $\beta$ of $B$, the Frobenius homomorphism $f: A \rightarrow B$, and the dual bases of $A$ over $B$ with respect to $f$ and $\beta$. We also discuss the notion of extensions of "integral type"; this definition, from [Sch 92], basically says that a finite extension $B \subset A$ of Hopf algebras has associated to it integral-like elements in $A$ and in $\bar{A}^{*}$, where $\bar{A}=A / A B^{+}$. These elements behave as though $\bar{A}$ were a finite-dimensional Hopf algebra. The existence of such elements is crucial in showing that an extension is $\beta$-Frobenius.

In Section 2 we consider a more general situation to which our results apply: that of bi-Galois extensions. Our main example of this set-up is the following: assume that $B \subset A$ and $H$ are Hopf algebras, and $\pi: A \rightarrow H$ is a surjective Hopf algebra map such that $\pi$ restricted to $B$ is also surjective. Then $A$ is an $H$-comodule in the natural way, so we may define $R=A^{c o H}$ and $S=B^{c o H}$. This gives a bi-Galois extension for the pair $(W, H)$, where $W$ is the coalgebra $A / A B^{+}$; it is summarized in the diagram

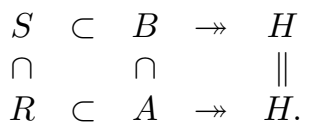

In the example of Hopf algebras $S \subset R$ in the Yetter-Drinfeld category ${ }_{H}^{H} \mathcal{Y D}$, we have $B=S \star H$ and $A=R \star H$. In a more general bi-Galois extension, $B$ and $A$ are not themselves Hopf algebras, but rather bicomodule algebras with respect to another pair of Hopf algebras.

The main result of Section 3 is that under suitable conditions (including a certain Hopf extension being of integral type), the property of being a $\beta$-Frobenius extension is inherited by the subalgebras of coinvariants. That is, if the pair of algebras $B \subset A$ in a bi-Galois extension is $\beta$-Frobenius then the subalgebras of coinvariants $S \subset R$ will also be $\beta$-Frobenius. This will be applied to the case of Hopf algebras in categories in Section 5 .

Section 4 is concerned with conditions under which extensions are of integral type. In fact we consider a more general situation: we look at extensions $K \subset H$, where $H$ is a Hopf algebra but $K$ is only a right coideal subalgebra. We prove (Theorem 4.8) that for $\bar{H}=H / H K^{+}$, the extension $K \subset H$ is of integral type provided $\operatorname{dim} \bar{H}<\infty$ and $H$ has a (right) normal basis over $K$; moreover, $\bar{H}^{*}$ is a Frobenius algebra. As a consequence we show that an extension of Hopf algebras $K \subset H$ is $\beta$-Frobenius if the coradical of $H$ is cocommutative and $\bar{H}$ is finite dimensional (Corollary 4.9). We also show (Theorem 4.10) that if $\operatorname{dim} K<\infty$ and $H$ has a right normal basis over $K$, then $K$ is a Frobenius algebra. This is applied to prove a Maschke-type theorem for (left) coideal subalgebras.

Section 5 studies Hopf algebras in the Yetter-Drinfeld category ${ }_{H}^{H} \mathcal{Y D}$. Our main result, Theorem 5.6, has already been mentioned above; in addition we also give a description of the automorphism $\beta$ of $S$, the Frobenius map $f: R \rightarrow S$, and the dual bases of $R$ over $S$. We also show that any finite-dimensional Hopf algebra $R$ in ${ }_{H}^{H} \mathcal{Y D}$ behaves like an ordinary Hopf algebra in that it is always a Frobenius algebra and satisfies a Maschke-type theorem: $R$ is semisimple $\Leftrightarrow \varepsilon(t) \neq 0$, for $t$ an integral in $R$ (Corollary 5.8). An important example of Hopf algebras in ${ }_{H}^{H} \mathcal{Y D}$ is given by $G$-graded Hopf algebras. We discuss an old example of Radford in this context, and explicitly compute various data for it such as the integral and dual bases. 
Finally in Section 6 we give our application to enveloping algebras of Lie coloralgebras. The results of Section 5 apply, since for a $G$-Lie coloralgebra $L$, where $G$ is an abelian group with a given bicharacter, the enveloping algebra $U(L)$ is a $G$-graded Hopf algebra, and so is a Hopf algebra in the Yetter-Drinfeld category for $H=k G$.

We fix some notation. Throughout we work with algebras over a field $k$. For a Hopf algebra $H$, we denote the comultiplication $\triangle: H \rightarrow H \otimes H$ by $h \mapsto$ $\sum h_{1} \otimes h_{2} . \quad H$ has counit $\varepsilon: H \rightarrow k$ and antipode $S: H \rightarrow H$. When $S$ is (composition) invertible, we denote its inverse by $\bar{S}$. Although sometimes $S$ also denotes a subring, the meaning should be clear from the context. Let $A$ be a right $H$-comodule, via $\delta: A \rightarrow A \otimes H, a \mapsto \sum a_{0} \otimes a_{1}$. If $\pi: H \rightarrow \bar{H}$ is a coalgebra surjection, then $A$ is also a right $\bar{H}$-comodule in the natural way, via $\bar{\delta}=(i d \otimes \pi) \circ \delta$. In this situation we say that $A$ has the induced $\bar{H}$-comodule structure.

Recall that for any algebra $A$, there is a left (right) action of $A$ on its dual $A^{*}$ given by the transpose of right (left) multiplication of $A$ on itself. As in [Sw], we write $a \rightarrow f$ for this left action and $f \leftarrow a$ for the right action, for all $a \in A, f \in A^{*}$. Equivalently $(a-f)(b)=f(b a)$ and $(f \leftarrow a)(b)=f(a b)$, for all $b \in A$. When $f$ is in the coalgebra $A^{\circ} \subseteq A^{*}$, we have the usual formulas $a \rightarrow f=\sum f_{2}(a) f_{1}$ and $f<a=\sum f_{1}(a) f_{2}$.

If $A$ is any augmented algebra with augmentation $\varepsilon: A \rightarrow k$, one can define (left

and right) integrals for $A$. That is, $\int_{A}^{l}=\{t \in A \mid a t=\varepsilon(a) t$, all $a \in A\}$ is the space of left integrals; similarly for $\int_{A}^{r}$, the right integrals. If $A$ is a Frobenius algebra, then the spaces of left and right integrals are each one-dimensional. In this case, if $f \in A^{*}$ is the Frobenius homomorphism for $A$ and we choose $t \in A$ such that $f<t=\varepsilon$, then it is easy to see that $t \in \int_{A}^{r}$. If also $A^{*}$ is augmented then $f \in \int_{A^{*}}^{r}$.

Finally, we call an extension of algebras $B \subset A$ faithfully flat if $A$ is a faithfully flat left and right $B$-module. When $A$ and $B$ are Hopf algebras and the antipode is bijective, assuming faithful flatness on one side is sufficient, since $S$ is then an antiautomorphism.

\section{1. $\beta$-Frobenius EXtensions}

We begin by reviewing some known results about $\beta$-Frobenius extensions, with some additional facts and characterizations which will be used later. $\beta$-Frobenius extensions generalize classical Frobenius extensions $[\mathrm{K}]$ and were introduced by Nakayama and Tsuzuku [NT 60], [NT 61]; they are also called Frobenius extensions of the second kind. Recall the definition:

1.1. Definition. Let $B \subset A$ be a ring extension and $\beta: B \rightarrow B$ a ring automorphism.

(a) If $M$ is a left $B$-module then ${ }_{\beta} M$ is defined to be the left $\beta$-twisted $B$-module with underlying set $M$ and left action $b \cdot \beta=\beta(b) \cdot m$ for all $m \in M, b \in B$.

Similarly, one can define a right $\beta$-twisted $B$-module.

(b) $B \subset A$ is called a (left) $\beta$-Frobenius extension if

(i) $A$ is a finitely generated projective right $B$-module, and

(ii) $A \cong{ }_{\beta} \operatorname{Hom}_{B}\left(A_{B}, B_{B}\right)$ as $(B, A)$-bimodules, where ${ }_{\beta} \operatorname{Hom}_{B}\left(A_{B}, B_{B}\right)$ is a left $\beta$-twisted $(B, A)$-bimodule via $(b \cdot \psi \cdot a)(x)=\beta(b) \psi(a x)$, for all $b \in B, a, x \in A$, and $\psi \in \operatorname{Hom}_{B}\left(A_{B}, B_{B}\right)$. 
1.2. Remark. (a) In the case when $\beta=i d$ we recover the classical notion of a Frobenius extension. If also $B=k$, then $A$ is a Frobenius algebra since the isomorphism $A_{A} \rightarrow\left(A^{*}\right)_{A}$ of part (ii) of the definition is the classical Frobenius isomorphism.

(b) It will follow from Proposition 1.3 (c) that the choice of sides for a $\beta$-Frobenius extension does not matter; any (left) $\beta$-Frobenius extension is also a (right) $\beta^{-1}$ Frobenius extension. Note also that for any $B$-modules $X_{B}$ and ${ }_{B} Y$, we have

$$
X_{\beta} \otimes_{B} Y \cong X \otimes_{B} \beta^{-1} Y
$$

as vector spaces, via $x \otimes y \mapsto x \otimes y$.

As in the classical case, dual bases exist for a $\beta$-Frobenius extension. In fact, these concepts are equivalent, as we show next. A similar result is shown in [BF, Theorem 1.1]; see Remark 1.4 (a).

1.3. Proposition. Let $B \subset A$ be a ring extension, $\beta: B \rightarrow B$ a ring automorphism, and $f: A \rightarrow{ }_{\beta} B$ any $(B, B)$-bimodule map. Define

$$
\begin{array}{ll}
F: A \rightarrow \operatorname{Hom}_{B}\left(A_{B}, B_{B}\right) & \text { by } F(x)=f x, \text { where } f x(a)=f(x a) ; \\
\tilde{F}: A_{\beta} \otimes_{B} A \rightarrow \operatorname{Hom}_{B}\left(A_{B}, A_{B}\right) & \text { by } \tilde{F}(x \otimes y)=x f y=x F(y), \text { where } \\
& x F(y)(a)=x f(y a)
\end{array}
$$

for all $x, y \in A$. Then the following are equivalent:

(a) $B \subset A$ is a $\beta$-Frobenius extension via $F$.

(b) $F$ and $\tilde{F}$ are bijections.

(c) There exist $r_{i}, l_{i} \in A, i=1, \ldots, n$, such that $\forall a \in A$,

(i) $a=\sum_{i=1}^{n} r_{i} f\left(l_{i} a\right)$,

(ii) $a=\sum_{i=1}^{n}\left(\beta^{-1} \circ f\right)\left(a r_{i}\right) l_{i}$.

Proof. $(a) \Rightarrow(b)$. First note that $F$ is indeed a map of $(B, A)$-bimodules and $\tilde{F}$ is well defined. Next, $F$ is bijective by the definition of a $\beta$-Frobenius extension; to see that $\tilde{F}$ is bijective, we note that $\tilde{F}$ is defined via $F$ as follows:

$$
\begin{aligned}
A_{\beta} \otimes_{B} A & \stackrel{\operatorname{id} \otimes F}{\longrightarrow} A_{\beta} \otimes_{B}{ }_{\beta} \operatorname{Hom}_{B}\left(A_{B}, B_{B}\right) \\
& \cong A \otimes_{B} \operatorname{Hom}_{B}\left(A_{B}, B_{B}\right) \quad \text { by } 1.2(\mathrm{~b}) \\
& \cong \operatorname{Hom}_{B}\left(A_{B},\left(A \otimes_{B} B\right)_{B}\right) \\
& \cong \operatorname{Hom}_{B}\left(A_{B}, A_{B}\right) .
\end{aligned}
$$

For, following the isomorphisms, for any $x, y \in A$ we have $x \otimes y \mapsto x \otimes F(y)=$ $x \otimes f y \mapsto x \otimes f y$. This now goes to the map $a \mapsto x f(y a)$, which is precisely $\tilde{F}(x \otimes y)$.

$(b) \Rightarrow(c)$. Since $\tilde{F}: A_{\beta} \otimes_{B} A \rightarrow \operatorname{Hom}_{B}\left(A_{B}, A_{B}\right)$ is bijective, we may choose $\sum_{i=1}^{n} r_{i} \otimes l_{i} \in A_{\beta} \otimes_{B} A$ such that $\tilde{F}\left(\sum r_{i} \otimes l_{i}\right)=i d_{A}$. But then

$$
\sum r_{i} f\left(l_{i} a\right)=\tilde{F}\left(\sum r_{i} \otimes l_{i}\right)(a)=a,
$$

so (i) holds.

As for (ii), let $x, y \in A$. Then

$$
\begin{aligned}
& F\left(\sum\left(\beta^{-1} \circ f\right)\left(x r_{i}\right) l_{i}\right)(y)=f\left(\sum \beta^{-1}\left(f\left(x r_{i}\right)\right) l_{i} y\right) \\
& \quad=\sum f\left(x r_{i}\right) f\left(l_{i} y\right)=f\left(x \sum r_{i} f\left(l_{i} y\right)\right)=f(x y)=F(x)(y) .
\end{aligned}
$$

Bijectivity of $F$ now yields (ii). 
$(c) \Rightarrow(a) . A_{B}$ is finitely generated by (i), using the dual basis lemma. Moreover, $A \cong{ }_{\beta} \operatorname{Hom}_{B}\left(A_{B}, B_{B}\right)$ via the inverse bijections:

$$
x \stackrel{F}{\mapsto} f x \quad \text { and } \quad \sum \beta^{-1}\left(\psi\left(r_{i}\right)\right) l_{i} \stackrel{G}{\leftrightarrow} \psi,
$$

using (i) to see that $F \circ G=i d$ and (ii) to see that $G \circ F=i d$. It is easy to see that $F$ is indeed a $(B, A)$-bimodule map.

1.4. Remark. (a) Any $f: A \rightarrow B$ satisfying part (c) is called a $\beta$-Frobenius homomorphism, and $\left\{r_{i}\right\},\left\{l_{i}\right\}$ are a pair of dual bases. The Frobenius homomorphism $f$ determines a $B$-semi-linear associative form ( , ) : $A \otimes A \rightarrow B$ via $\left(a, a^{\prime}\right):=f\left(a a^{\prime}\right)$, and it follows from 1.3 (c) that this form is nondegenerate. Here "semi-linear" means that for $b, b^{\prime} \in B,\left(b a, a^{\prime} b^{\prime}\right)=\beta(b)\left(a, a^{\prime}\right) b$. Conversely the existence of such a form determines an isomorphism $F: A \rightarrow \operatorname{Hom}(A, B)$ given by $F x(a):=(x, a)$. This characterization of $\beta$-Frobenius is the one given in [BF, 1.1].

(b) In the classical case of a Frobenius algebra A, one may define the Nakayama automorphism $\eta$ of $A$ by $f(x y)=f(y \eta(x))$, for all $x, y \in A$. Equivalently $(x, y)=$ $(y, \eta(x))$, where the bilinear form is as in (a).

(c) When $B \subset A$ is a $\beta$-Frobenius extension as above, $A$ satisfies the separability condition

$$
\sum a r_{i} \otimes l_{i}=\sum r_{i} \otimes l_{i} a
$$

in the twisted tensor product $A_{\beta} \otimes_{B} A$, for all $a \in A$. For, given $a, x \in A$,

$$
\tilde{F}\left(\sum a r_{i} \otimes l_{i}\right)(x)=\sum a r_{i} f\left(l_{i} x\right)=a x=\sum r_{i} f\left(l_{i} a x\right)=\tilde{F}\left(\sum r_{i} \otimes l_{i} a\right)(x) ;
$$

the bijectivity of $\tilde{F}$ now yields the result.

(d) If $B \subset A$ are finite-dimensional and $A_{B}$ is free, then (c) (ii) in the proposition is not needed; that is, $B \subset A$ is $\beta$-Frobenius if there exist a $(B, B)$-bimodule map $f: A \rightarrow \beta B$ and $\left\{r_{i}, l_{i}\right\}$ in $A$ such that $a=\sum r_{i} f\left(l_{i} a\right)$, for all $a$. For, by the comment in the proof of $(c) \Rightarrow(a)$, (i) implies $F \circ G=i d$, and thus $F$ is surjective. Since $A \cong B^{(n)}$ for some $n$, it follows that $\operatorname{Hom}_{B}(A, B) \cong \operatorname{Hom}_{B}(B, B)^{(n)} \cong B^{(n)}$ and so $\operatorname{dim} A=\operatorname{dim} \operatorname{Hom}_{B}(A, B)$. Thus $F$ is bijective, and so $B \subset A$ is $\beta$-Frobenius.

A major example of $\beta$-Frobenius extensions is given by any pair $B \subset A$ of finite-dimensional Hopf algebras; this is essentially [Sch 92, $3.6 \mathrm{II}]$. We give here a shorter proof of this fact, and also obtain some new information about the form of the automorphism $\beta$, the map $f$, and the dual bases.

We fix the following notation. Let $A$ be a finite-dimensional Hopf algebra, $f_{A}$ a right integral in $A^{*}$, and $t \in A$ such that $f_{A}<t=\varepsilon$ (that is, $f_{A}(t a)=\varepsilon(a)$, for all $a \in A$ ). We may choose $t$ in this way since by the Larson-Sweedler theorem [LS] $f_{A}$ is a generator for $A^{*}$ as a (cyclic) right $A$-module. As noted in the introduction, it follows that $t \in \int_{A}^{r}$. Let $\alpha \in A^{*}$ be the (right) modular function for $A$; that is, at $=\alpha(a) t$, for all $a \in A$.

Parts of the next lemma are known: the fact that $A$ is Frobenius is in [LS], and the form of the dual bases and the Nakayama automorphism $\eta$ were shown in [OSch] under an additional hypothesis.

1.5. Lemma. Let $A$ be any finite-dimensional Hopf algebra with $f_{A}, t$, and $\alpha$ as above. Then $A$ is a Frobenius algebra with Frobenius homomorphism $f_{A}$ and dual 
bases $\left\{\bar{S} t_{2}, t_{1}\right\}$. The Nakayama automorphism $\eta: A \rightarrow A$ has the following form:

$$
\begin{aligned}
& \eta(a)=\bar{S}^{2}(a \leftarrow \alpha)=\left(\bar{S}^{2} a\right) \leftarrow \alpha, \\
& \eta^{-1}(a)=S^{2}\left(a \leftarrow \alpha^{-1}\right)=\left(S^{2} a\right) \leftarrow \alpha^{-1}
\end{aligned}
$$

for all $a \in A$. It follows that $\eta$ has finite order dividing $2 \operatorname{dim}_{k} A$.

Proof. Since $f_{A}$ is a right integral of $A^{*}, a \leftarrow f_{A}=f_{A}(a) 1_{A}$, for all $a \in A$. It follows that

$$
\begin{aligned}
\sum\left(\bar{S} t_{2}\right) f_{A}\left(t_{1} a\right) & =\sum\left(\bar{S} t_{3}\right) f_{A}\left(t_{1} a_{1}\right) t_{2} a_{2} \\
& =\sum f_{A}\left(t a_{1}\right) a_{2}=\sum \varepsilon\left(a_{1}\right) a_{2}=a,
\end{aligned}
$$

and thus $\left\{\bar{S} t_{2}, t_{1}\right\}$ are dual bases of $A$ and $f_{A}$ is the Frobenius homomorphism.

Now replace $a$ by $\eta(a)$ in the above formula:

$$
\eta(a)=\sum\left(\bar{S} t_{2}\right) f_{A}\left(t_{1} \eta(a)\right)=\sum\left(\bar{S} t_{2}\right) f_{A}\left(a t_{1}\right),
$$

since $f_{A}$ is the Frobenius homomorphism. Thus

$$
\begin{aligned}
S^{2}(\eta(a)) & =\sum f_{A}\left(a t_{1}\right)\left(S t_{2}\right)=\sum f_{A}\left(a_{1} t_{1}\right) a_{2} t_{2}\left(S t_{3}\right) \\
& =\sum f_{A}\left(a_{1} t\right) a_{2}=\sum \alpha\left(a_{1}\right) a_{2}=a-\alpha .
\end{aligned}
$$

Hence $\eta(a)=\bar{S}^{2}(a \leftarrow \alpha)$. Since $\alpha \bar{S}^{2}=\alpha$, it follows that $\eta(a)=\left(\bar{S}^{2} a\right)-\alpha$. Similarly, using $\alpha S=\alpha^{-1}$ and $\alpha S^{2}=\alpha$, it follows that $\eta^{-1}(a)=S^{2}\left(a \leftarrow \alpha^{-1}\right)=$ $\left(S^{2} a\right) \leftarrow \alpha^{-1}$, for all $a \in A$.

We now consider the order of $\eta$. First, for $a \in A$,

$$
\begin{aligned}
\eta^{2}(a) & =\bar{S}^{2}\left(\left(\bar{S}^{2} a\right) \leftarrow \alpha\right) \leftarrow \alpha \\
& =\left(\bar{S}^{4} a\right)-\alpha^{2} .
\end{aligned}
$$

By induction it follows that $\eta^{n}(a)=\left(\bar{S}^{2 n} a\right)-\alpha^{n}$. Since $\alpha \in G\left(A^{*}\right)$, the order of $\alpha$ divides $n=\operatorname{dim} A[\mathrm{NZ}]$ and so $\eta^{n}(a)=\bar{S}^{2 n}(a)$; similarly the order of the modular function for $A^{*}, c \in A^{* *} \cong A$, divides $n$. Since $S^{4}(a)=c\left(\alpha^{-1} \rightarrow a \leftarrow \alpha\right) c^{-1}$ by Radford's therorem [R 76], it follows that $S^{4 n}=i d$. Thus $\eta^{2 n}(a)=\bar{S}^{4 n}(a)=a$, and so the order of $\eta$ divides $2 n$.

We remark that as a consequence of Lemma $1.5, f_{A}(\bar{S} t)=1$. For, set $a=\bar{S} t$ in the dual basis formula in the proof. Since $\bar{S} t \in \int_{A}^{l}$, this gives $\bar{S} t f_{A}(\bar{S} t)=\bar{S} t$, and so $f_{A}(\bar{S} t)=1$.

Now assume that $B$ is a Hopf subalgebra of $A$; let $t_{B}$ be a right integral in $B$ and let $\alpha_{B} \in B^{*}$ be the right modular function for $B$. Since $A$ is free over $B$, by [NZ], we may write

$$
t=t_{A}=\tilde{\Lambda} t_{B}
$$

for some $\tilde{\Lambda} \in A$.

1.6. Definition. Let $B \subset A$ be finite-dimensional Hopf algebras, with right modular functions $\alpha_{A}$ and $\alpha_{B}$ and Nakayama automorphisms $\eta_{A}$ and $\eta_{B}$ respectively. Let

and

$$
\chi=\alpha_{A} * \alpha_{B}^{-1} \in \operatorname{Alg}(B, k)
$$

$$
\beta=\eta_{B}^{-1} \circ \eta_{A} \in \operatorname{Aut}(B),
$$


where here $\alpha_{A}$ and $\eta_{A}$ are the respective restrictions to $B$. Then $\chi$ is the relative modular function and $\beta$ the relative Nakayama automorphism.

It is easy to see that the Nakayama automorphism of $A$ restricts to an automorphism of $B$ and that $\beta(b)=\Sigma \chi\left(b_{1}\right) b_{2}=b \leftarrow \chi$, using the formulas in Lemma 1.5.

1.7. Theorem. Let $B \subset A$ be finite-dimensional Hopf algebras, and consider $A$ as a left $\bar{A}=A / A B^{+}$-comodule via the induced coaction. Choose $f_{A} \in \int_{A^{*}}^{r}$ and $t_{A} \in \int_{A}^{r}$ such that $f_{A} \leftarrow t_{A}=\varepsilon$. Let $t_{B} \in \int_{B}^{r}$, and write $t_{A}=\tilde{\Lambda} t_{B}$. Then $B \subset A$ is a $\beta$-Frobenius extension, with

(a) automorphism $\beta: B \rightarrow B$ given by $\beta=\eta_{B}^{-1} \circ \eta_{A}$, the relative Nakayama automorphism as in 1.6,

(b) Frobenius homomorphism $f: A \rightarrow B$, via $f(a)=\sum f_{A}\left(a_{1} \bar{S} t_{B}\right) a_{2}$,

(c) dual bases $\left\{\bar{S} \Lambda_{2}, \Lambda_{1}\right\}$, where $\Lambda=(S \tilde{\Lambda}) \leftarrow \alpha^{-1}=\eta^{-1}(\bar{S} \tilde{\Lambda})$.

Proof. We first note several consequences of the fact that $A$ is free over $B$ [NZ]. Setting $\bar{A}=A / A B^{+}$, we may consider $A$ as a left $\bar{A}$-comodule in the natural way; it follows that $B={ }^{c o} \bar{A} A$ (as in 2.4 (b)). Also by Remark 1.4 (d), it will suffice to show that the given dual bases satisfy 1.3 (c) (i).

The fact that $B={ }^{c o} \bar{A} A$ implies that $\operatorname{Im} f \subseteq B$. For, the comodule map $\rho: A \rightarrow \bar{A} \otimes A$ is given by $\rho=(\pi \otimes i d) \circ \triangle$. Writing $u=\bar{S} t_{B}$, we have:

$$
\begin{aligned}
\rho(f(a)) & =(\pi \otimes i d) \circ \triangle\left(\sum f_{A}\left(a_{1} u\right) a_{2}\right) \\
& =\sum f_{A}\left(a_{1} u\right) \bar{a}_{2} \otimes a_{3} \\
& =\sum f_{A}\left(a_{1} u_{1}\right) \bar{a}_{2} \varepsilon\left(u_{2}\right) \otimes a_{3} \\
& =\sum f_{A}\left(a_{1} u_{1}\right) \overline{a_{2} u_{2}} \otimes a_{3} \quad \text { since } u_{2} \in B \\
& =\sum \frac{a_{1} u \leftarrow f_{A}}{a_{1}} \otimes a_{2} \\
& =\sum f_{A}\left(a_{1} u\right) \overline{1} \otimes a_{2} \quad \text { since } f_{A} \in \int_{A^{*}}^{r} \\
& =\overline{1} \otimes f(a) .
\end{aligned}
$$

Thus $f(a) \in B$.

Since $u \in \int_{B}^{l}$, for all $b \in B$ we have

$$
f(a b)=\sum f_{A}\left(a_{1} b_{1} u\right) a_{2} b_{2}=\sum f_{A}\left(a_{1} \varepsilon\left(b_{1}\right) u\right) a_{2} b_{2}=f(a) b
$$

and so $f$ is a right $B$-map. On the left, using the Nakayama automorphism $\eta_{A}$,

$$
\begin{aligned}
f(b a) & =\sum f_{A}\left(b_{1} a_{1} u\right) b_{2} a_{2}=\sum f_{A}\left(a_{1} u \eta_{A}\left(b_{1}\right)\right) b_{2} a_{2} \\
& =\sum f_{A}\left(a_{1} u \alpha_{B}^{-1}\left(\eta_{A}\left(b_{1}\right)\right)\right) b_{2} a_{2} \\
& =\sum \alpha_{B}^{-1}\left(\bar{S}^{2}\left(b_{1} \leftarrow \alpha_{A}\right)\right) b_{2} f_{A}\left(a_{1} u\right) a_{2} \quad \text { by Lemma } 1.5 \\
& =\sum \alpha_{A}\left(b_{1}\right) \alpha_{B}^{-1}\left(b_{2}\right) b_{3} f(a) \\
& =\sum \chi\left(b_{1}\right) b_{2} f(a)=\beta(b) f(a) .
\end{aligned}
$$

Thus $f$ is a left $\beta$-twisted $B$-map. 
It remains only to check 1.3 (c) (i) for the dual bases $\left\{\bar{S} \Lambda_{2}, \Lambda_{1}\right\}$, where $\eta(\Lambda)=\bar{S} \tilde{\Lambda}$ and $t_{A}=\tilde{\Lambda} t_{B}$. Now for $a \in A$,

$$
\begin{aligned}
\sum\left(\bar{S} \Lambda_{2}\right) f\left(\Lambda_{1} a\right) & =\sum\left(\bar{S} \Lambda_{3}\right) f_{A}\left(\Lambda_{1} a_{1} \bar{S} t_{B}\right) \Lambda_{2} a_{2} \\
& =\sum f_{A}\left(\Lambda_{1} a_{1} \bar{S} t_{B}\right) \varepsilon\left(\Lambda_{2}\right) a_{2} \\
& =\sum f_{A}\left(\Lambda a_{1} \bar{S} t_{B}\right) a_{2} \\
& =\sum f_{A}\left(a_{1} \bar{S} t_{B} \eta(\Lambda)\right) a_{2} \\
& =\sum f_{A}\left(a_{1} \bar{S}\left(\tilde{\Lambda} t_{B}\right)\right) a_{2} \quad \text { by the form of } \eta(\Lambda) \\
& =\sum f_{A}\left(a_{1} \bar{S} t_{A}\right) a_{2} \\
& =\sum f_{A}\left(\varepsilon\left(a_{1}\right) \bar{S} t_{A}\right) a_{2} \quad \text { since } \bar{S} t_{A} \in \int_{A}^{l} \\
& =f_{A}\left(\bar{S} t_{A}\right) a=a
\end{aligned}
$$

since $f_{A}\left(\bar{S} t_{A}\right)=1$ by choice of $t_{A}$ with respect to $f_{A}$, as noted after Lemma 1.5. Thus $B \subset A$ is $\beta$-Frobenius.

1.8. Corollary. Let $B \subset A$ be finite-dimensional Hopf algebras. Then the following are equivalent:

(a) $B \subset A$ is a classical Frobenius extension;

(b) $\beta=i d$, where $\beta$ is the relative Nakayama automorphism as in 1.6;

(c) $\left.\alpha_{A}\right|_{B}=\alpha_{B}$, where $\alpha_{A}$ and $\alpha_{B}$ are the right modular functions for $A$ and $B$, respectively.

Proof. $(c) \Rightarrow(b)$ by the form of $\chi$ in Definition 1.6, and $(b) \Rightarrow(a)$ is trivial. Thus it remains to prove $(a) \Rightarrow(c)$. The argument is the same as that in [OSch, 4.8, $(2) \Rightarrow(3)]$ : since $A$ is both Frobenius and $\beta$-Frobenius, there exists an isomorphism $\phi: A \rightarrow \beta^{-1} A$ from $A$ as an ordinary $(B, A)$-module to $A$ as a left $\beta^{-1}$-twisted $(B, A)$-module. Let $u=\phi(1)$; then $u$ is a unit in $A$, with inverse $w=\phi^{-1}(1)$. It follows that for all $b \in B$,

$$
u b=\phi(1) b=\phi(b)=\beta^{-1}(b) \phi(1)=\beta^{-1}(b) u .
$$

Applying $\varepsilon$, we see that $\varepsilon(b)=\varepsilon\left(\beta^{-1}(b)\right)$, for all $b$, since $\varepsilon(u) \neq 0$. Thus $\varepsilon \beta=\varepsilon$. But since $\beta(b)=\sum \chi\left(b_{1}\right) b_{2}$, it follows that $\varepsilon \beta=\chi$. Thus $\chi=\varepsilon$. Now by 1.6 again, the restriction of the modular function of $A$ to $B$ is the modular function of $B$ : $\left.\alpha\right|_{B}=\alpha_{B}$.

We remark that this result is reminiscent of a classical result on locally compact groups [We, Ch. II, Sec. 9]: if $G$ is such a group and $L$ a closed subgroup of $G$, consider the locally compact topological space $X=G / L$ of left cosets of $L$ in $G$; $G$ acts on $X$ by left translation. Then $X$ has a non-zero $G$-invariant Radon measure $\Leftrightarrow$ the modular function for $G$ restricted to $L$ is the modular function for $L$.

1.9. Example. Let $H$ be any finite-dimensional Hopf algebra and $D(H)$ its Drinfeld double [Dr 86]. Following the relations for $D(H)$ given in [R 93], we may write $D(H)=H^{* c o p} \bowtie H$; thus $D(H)=H^{* c o p} \otimes H$ as coalgebras, and the multiplication is given by $H^{* c o p}$ and $H$ acting on each other via the right and left coadjoint actions. See also $[\mathrm{M}, 10.3 .5]$ for details. In particular we may consider $H \subset D(H)$, via $H \cong \varepsilon \bowtie H$, and apply the above results to this extension, which is always $\beta$-Frobenius by Theorem 1.7.

By [R 93], $D(H)$ is always unimodular, and thus $\alpha=\varepsilon$ in $A=D(H)$. Consequently, for $\chi$ as in Definition 1.6 and Theorem 1.7, $\chi=\left(\alpha_{H}\right)^{-1}$, where $\alpha_{H}$ is the 
right modular function for $B=H$. It follows that the automorphism $\beta$ is given by

$$
\beta(h)=\sum \alpha_{H}^{-1}\left(h_{1}\right) h_{2}
$$

for all $h \in H$. Thus, as in Corollary 1.8, the extension $H \subset D(H)$ is Frobenius $\Leftrightarrow \alpha_{H}=\varepsilon \Leftrightarrow H$ is unimodular. This last fact is also noted in [CMZ, Cor.4.5]. We may also describe the Frobenius homomorphism $f$ as follows. Using Theorem 1.7(b) and Example 1.12, we see that $f(a)=\sum \lambda\left(\overline{a_{1}}\right) a_{2}$, where $\lambda \in \int_{\overline{D(H)}}^{r}$. Now, $\overline{D(H)}=$ $D(H) / D(H) H^{+} \cong H^{* c o p}$ as coalgebras, and thus a right integral $\lambda$ in $\overline{D(H)}^{*} \cong H^{o p}$ corresponds to a left integral in $H$. That is, we may write $\lambda=u \in \int_{H}^{l}$. Then for an element $\gamma \bowtie h \in D(H)$, where $\gamma \in H^{* c o p}, h \in H$, we have $\lambda(\overline{\gamma \bowtie h})=$ $\lambda(\overline{\gamma \bowtie \varepsilon(h) 1})=\varepsilon(h) \gamma(u)$, and thus

$$
\begin{aligned}
f(\gamma \bowtie h) & :=\sum \lambda\left(\overline{\gamma_{2} \bowtie h_{1}}\right)\left(\gamma_{1} \bowtie h_{2}\right) \\
& =\sum \gamma_{2}(u) \gamma_{1} \bowtie h \\
& =\sum(u \rightarrow \gamma) \bowtie h=\gamma(u) \varepsilon \bowtie h .
\end{aligned}
$$

Thus in fact $f: D(H) \rightarrow \varepsilon \bowtie H \cong H$; one may check that $f$ is a left $\beta$-twisted map.

We may also find a dual basis for $D(H)$ over $H$. For, let $\tilde{\Lambda}$ be a left integral in $H^{*}{ }^{c o p}$ and $t_{H}$ a right integral in $H$. Then $t=\tilde{\Lambda} \bowtie t_{H}$ is a (left and right) integral in $D(H)$ [R 93]. Since $\alpha=\varepsilon$ in $D(H)$, Theorem 1.7 (c) gives that $\Lambda=S \tilde{\Lambda}$. Thus the dual bases are $\left\{\bar{S} \Lambda_{2}, \Lambda_{1}\right\}=\left\{\tilde{\Lambda}_{1}, S \tilde{\Lambda}_{2}\right\}$.

We next turn to studying more general conditions which will guarantee that a given extension $B \subset A$ is $\beta$-Frobenius. Although we may no longer have actual integrals in $A$ and $B$, the hypotheses we use involve the existence of integral-like elements, as used in [Sch 92]. If $A$ is any augmented algebra with augmentation $\varepsilon: A \rightarrow k$, let $\int_{A}^{r}$ (respectively $\int_{A}^{l}$ ) denote the space of right (left) integrals of $A$.

1.10. Definition. Let $W$ be a Hopf algebra, $U \subset W$ a Hopf subalgebra, and $\bar{W}=W / W U^{+}$the (left) quotient coalgebra; note that $\bar{W}^{*}$ is an augmented algebra by evaluation at $\overline{1}$. Then the extension $U \subset W$ is of (right) integral type if

(a) there exists $0 \neq \lambda \in \int_{\bar{W}^{*}}^{r}$,

(b) there exists $\Lambda \in W$ such that $\lambda \leftarrow \Lambda=\varepsilon$ on $\bar{W}$,

(c) there exists $\chi \in \operatorname{Alg}(U, k)$ such that $\lambda \leftarrow u=\chi(u) \lambda$, for all $u \in U$.

Note that (b) means that $\lambda(\Lambda \cdot \bar{w})=\lambda(\overline{\Lambda w})=\varepsilon(\bar{w})=\varepsilon(w)$, for all $w \in W$ (in particular $\lambda(\bar{\Lambda})=1)$; alternatively, $\int_{\bar{W}^{*}}^{r} \leftarrow W=\bar{W}^{*}$. Similarly (c) means that $\lambda(\overline{u w})=\chi(u) \lambda(\bar{w})$, for all $u \in U, w \in W$; or alternatively $\lambda \leftarrow U=k \lambda$.

Similarly we may define an extension $U \subset W$ to be of left integral type by using $\bar{W}=W / U^{+} W$ and assuming there exist $0 \neq \lambda \in \int_{\bar{W}^{*}}^{l}, \Lambda$, and $\chi$ with the appropriate properties. In either case the hypothesis generalizes properties of normal Hopf subalgebras.

1.11. Example. Let $U$ be a normal Hopf subalgebra of $W$ of finite index (that is, $\operatorname{dim} \bar{W}$ is finite). Then $U \subset W$ is of right and left integral type. For, since $U$ is normal, $U^{+} W=W U^{+}$and $\bar{W}=W / W U^{+}$is a finite-dimensional Hopf algebra. Thus $\bar{W}^{*}$ is also a finite-dimensional Hopf algebra, and so by the Larson-Sweedler theorem contains a right (left) integral $\lambda \neq 0$; moreover $\lambda \leftarrow \bar{W} \cong \bar{W}^{*}$. Thus there exists $\bar{\Lambda} \in \bar{W}$ such that $\lambda \leftarrow \bar{\Lambda}=\varepsilon$; in fact $\bar{\Lambda}$ is a right integral in $\bar{W}$. Choose 
$\Lambda \in W$ to be any pre-image of $\bar{\Lambda}$; then (b) holds. For (c), we may choose $\chi=\varepsilon$. For, if $u \in U$ and $w \in W$, then $\overline{u w}=\varepsilon(u) \bar{w}$ since $\overline{U^{+} W}=\overline{W U^{+}}=\overline{0}$. Thus $\lambda \leftarrow u=\varepsilon(u) \lambda$, for all $u \in U$.

In fact $U \subset W$ is always of (right and left) integral type if $W$ is finite-dimensional.

1.12. Example. We reconsider the case of finite-dimensional Hopf algebras of Theorem 1.7. It is not difficult to check that we may use $\lambda \in \bar{A}^{*}$ defined by $\lambda(\bar{a}):=f_{A}\left(a \bar{S} t_{B}\right)$, and that $\Lambda$ and $\chi$ are exactly as given in 1.7. Thus any such extension is of right (left) integral type. This fact was shown in [Sch 92], though without the explicit formulas for $\lambda, \Lambda$, and $\chi$.

1.13. Example. We show in Corollary 4.9 that any extension $B \subset A$ of pointed Hopf algebras of finite index is always of integral type.

The importance of integral-type extensions comes from the following result of Schneider. Recall that if $A$ is a left $W$-comodule via $\sigma: A \rightarrow W \otimes A$ and $\pi: W \rightarrow \bar{W}$ is a coalgebra morphism, then $A$ has an induced left $\bar{W}$-comodule structure via $(\pi \otimes i d) \circ \sigma$.

1.14. Theorem ([Sch 92, 3.3]). Let $W$ be a Hopf algebra with bijective antipode, let $U$ be a Hopf subalgebra such that $U \subset W$ is a faithfully flat extension of right integral type, and let $\bar{W}=W / W U^{+}$. Let $A$ be a left $W$-comodule algebra such that

${ }^{c o}{ }^{W} \subset A$ is $W$-Galois, and let $B={ }^{c o} \bar{W}_{A}$, where $A$ has the induced $\bar{W}$-comodule structure. Then $B \subset A$ is a $\beta$-Frobenius extension.

In particular the Frobenius homomorphism $f: A \rightarrow B$ is given by $f(a)=$ $\sum \lambda\left(\bar{a}_{-1}\right) a_{0}$, for all $a \in A$, and the automorphism $\beta: B \rightarrow B$ is given by $\beta(b)=$ $\sum \chi\left(\overline{b_{-1}}\right) b_{0}$, for all $b \in B$, where $\lambda$ and $\chi$ are as in Definition 1.10 .

In fact the result in [Sch 92] is stated for extensions of left integral type; the present version follows from that one by using $H:=W^{o p c o p}, H^{\prime}:=U^{o p c o p}$, and the right $H$-comodule algebra $A^{o p} \rightarrow A^{o p} \otimes W^{o p}{ }^{c o p}$ determined by $\sigma: A \rightarrow W \otimes A$.

\section{Bicomodule Algebras And Bi-Galois extensions}

Since we wish to show that in a Frobenius extension $B \subset A$, certain well-behaved subalgebras $S \subset R$ are also Frobenius, we introduce in this section the kind of extensions we will be looking at. We first consider a more general situation.

2.1. Definition. Let $C$ and $D$ be $k$-coalgebras and $A$ a $k$-vector space.

(1) $A$ is a left $(C, D)$-bicomodule if

(a) $A$ is a left $C$-comodule, with coaction $\sigma: A \rightarrow C \otimes A$,

(b) $A$ is a right $D$-comodule, with coaction $\rho: A \rightarrow A \otimes D$, and

(c) $\rho$ is a left $C$-comodule map (equivalently, $\sigma$ is a right $D$-comodule map).

That is, the following diagram commutes:

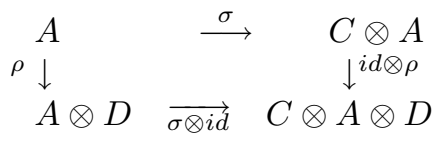

(2) Now assume that $C$ and $D$ have grouplike elements $c_{0}$ and $d_{0}$ respectively. Then we may define the coinvariants of $A$ with respect to these grouplikes to be

$$
{ }^{c o C} A=\left\{a \in A \mid \sigma(a)=c_{0} \otimes a\right\} \text { and } A^{c o D}=\left\{a \in A \mid \sigma(a)=a \otimes d_{0}\right\} .
$$

Then the set $S=\left({ }^{c o C} A\right) \cap\left(A^{c o D}\right)$ is the set of bicoinvariant elements. 
(3) If also $C$ and $D$ are bialgebras and $A$ is an algebra, then $A$ is a $(C, D)$ bicomodule algebra if $\sigma$ and $\rho$ are algebra maps.

2.2. Remark. (a) An easy example of a bicomodule is given as follows: Let $A$ be any coalgebra, and $C$ and $D$ two quotient coalgebras of $A$. Then $A$ becomes a right $D$-comodule and a left $C$-comodule via the induced coactions, and it is easy to see that $A$ is a $(C, D)$-bicomodule.

(b) If $a \in A$, then we may write $\sigma(a)=\sum a_{-1} \otimes a_{0} \in C \otimes A$ and $\rho(a)=$ $\sum a_{0} \otimes a_{1} \in A \otimes D$. This double usage of $a_{0}$ is justified since the compatibility condition 2.1 (c) allows us to write

$$
(i d \otimes \rho) \sigma(a)=(\sigma \otimes i d) \rho(a)=\sum a_{-1} \otimes a_{0} \otimes a_{1} .
$$

In the next two lemmas we relate the comodule structures of various subcomodules of coinvariants of a bicomodule $A$.

2.3. Lemma. Suppose $C$ and $D$ are coalgebras with grouplikes $c_{o}$ and $d_{0}$ (as in Definition 2.1(2)). Let $A$ be $a(C, D)$-bicomodule and set $B={ }^{c o} C A$ and $E=$ $A^{\text {co D }}$. Then:

(a) $B$ is a right $D$-comodule via $\rho$,

(b) $E$ is a left $C$-comodule via $\sigma$;

(c) set $S=B \cap E$; then $S=B^{\text {co } D}={ }^{c o} C E$.

Proof. (a) First note that $A \otimes D$ is a left $C$-comodule via $\sigma \otimes i d$. Moreover $B=$ ${ }^{\text {co } C} A$ implies that ${ }^{c o} C(A \otimes D)=B \otimes D$. Now if $b \in B$, then $\sigma(b)=c_{0} \otimes b$. By the bicomodule property,

$$
\begin{aligned}
(\sigma \otimes i d) \rho(b) & =(i d \otimes \rho) \sigma(b) \\
& =(i d \otimes \rho)\left(c_{0} \otimes b\right)=c_{0} \otimes \rho(b) .
\end{aligned}
$$

Thus $\rho(b) \in{ }^{c o C}(A \otimes D)=B \otimes D$.

(b) This is similar, using $(C \otimes A)^{c o} D=C \otimes E$.

(c) Since $E$ is a left $C$-comodule by (b), $B \cap E=\left({ }^{c o} C A\right) \cap E={ }^{c o} C E$. Also $B$ is a right $D$-comodule by (a), and so $B \cap E=B \cap\left(A^{\text {co } D}\right)=B^{\text {co } D}$.

We next require a known lemma [Sch 92, 1.3] although we sketch a proof for completeness, since we are working on the other side. Recall that if $W$ is a Hopf algebra, $U \subset W$ is a right coideal subalgebra if $U$ is a subalgebra and $\triangle U \subseteq U \otimes W$. It follows that $W U^{+}$is a coideal and a left ideal of $W$, and we have the canonical quotient map

$$
\mu: W \rightarrow \bar{W}:=W / W U^{+} .
$$

$\bar{W}$ is a coalgebra and a left $W$-module, and $W$ is a left $\bar{W}$-comodule via the induced coaction.

2.4. Lemma. Let $W$ be a Hopf algebra and $U \subset W$ a right coideal subalgebra such that $U \subset W$ is faithfully flat. Then

(a) the Galois map $\gamma: W \otimes_{U} W \rightarrow \bar{W} \otimes W$ given by $x \otimes y \mapsto \sum \bar{x}_{1} \otimes x_{2} y$ is bijective, and

(b) $U={ }^{c o} \bar{W} W$.

Proof. We follow the argument in [Sch 92, 1.3], switching from left to right.

(a) The map $\gamma$ is a bijection since it has the inverse $\bar{\gamma}: \bar{v} \otimes w \mapsto \sum v_{1} \otimes\left(S v_{2}\right) w$, for all $v, w \in W$, where here $S$ is the antipode in $W$. 
(b) This will follow from the commutativity of the diagram

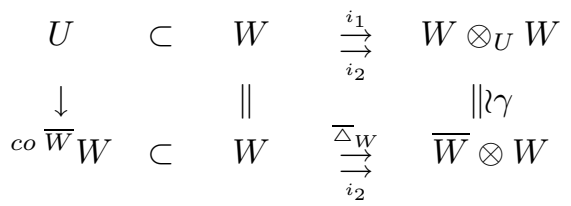

$x, y \in W$. The top row of the diagram is exact since $U \subset W$ is faithfully flat [Wa, Th.13.1], and the bottom row is also exact. But then $U=\operatorname{Ker}\left(\bar{\triangle}_{W}, i_{2}\right)=$ co $\bar{W} W$.

We now specialize to the following situation. Let $W$ and $H$ be Hopf algebras, $U$ a Hopf subalgebra of $W$, and let $\bar{W}$ be as above. If $A$ is a $(W, H)$-bicomodule algebra, then $A$ is a $(\bar{W}, H)$-bicomodule in the natural way, via $\bar{\sigma}=(\mu \otimes i d) \circ \sigma: A \rightarrow \bar{W} \otimes A$, and the $\bar{W}$-coinvariants ${ }^{c o \bar{W}} A$ are a subring of $A$ since $\bar{W}$ is a left $W$-module. From now on we use the notation

$$
R=A^{c o H}, B={ }^{c o} \bar{W} A, S=R \cap B .
$$

By Lemma 2.3, $S={ }^{c o} \bar{W} R=B^{c o H}$. We next show that these subrings are $U$-comodules.

2.5. Lemma. Assume $U \subset W$ is a faithfully flat extension of Hopf algebras, and let $\bar{W}, A, B, R$, and $S$ be as above. Then:

(a) $B$ is a left $U$-comodule via $\sigma$,

(b) $S$ is a left $U$-comodule via $\sigma$.

Proof. (a) We first show that $\sigma^{-1}(U \otimes A)=B$. Choose $a \in \sigma^{-1}(U \otimes A)$. Then $(\mu \otimes i d) \sigma(a)=\overline{1} \otimes a$, and thus $a \in{ }^{c o \bar{W}} A=B$. Hence $\sigma^{-1}(U \otimes A) \subseteq B$.

On the other hand, choose $b \in B$. By Lemma 2.4, $U=\operatorname{co} \bar{W} W$, and thus co $\bar{W}(W \otimes A)=U \otimes A$, where $W \otimes A$ is a $\bar{W}$-comodule via $\bar{\triangle}_{W} \otimes i d$. Now

$$
\begin{aligned}
\left(\bar{\triangle}_{W} \otimes i d\right) \sigma(b) & =\left(\mu \otimes i d^{2}\right)\left(\triangle_{W} \otimes i d\right) \sigma(b) \\
& =\left(\mu \otimes i d^{2}\right)(i d \otimes \sigma) \sigma(b) \text { since } \sigma \text { is a comodule map } \\
& =\sum \overline{b_{-1}} \otimes\left(b_{0}\right)_{-1} \otimes\left(b_{0}\right)_{0} \\
& =\overline{1} \otimes \sigma(b) \quad \text { since } b \in{ }^{c o} \bar{W} A .
\end{aligned}
$$

Thus $\sigma(b) \in{ }^{c o} \bar{W}(W \otimes A)=U \otimes A$, and so $b \in \sigma^{-1}(U \otimes A)$.

Now note that if $U \subset W$ is any inclusion of coalgebras and $A$ is a left $W$ comodule via $\sigma: A \rightarrow W \otimes A$, then $\sigma^{-1}(U \otimes A)$ is a left $U$-comodule. In our case this means that $B$ is a left $U$-comodule.

(b) Recall that $S=R \cap B$ by Lemma 2.3. The result now follows since $\sigma(S) \subset$ $\sigma(R) \subset W \otimes R$ by 2.3 (b) and since $\sigma(S) \subset \sigma(B) \subset U \otimes B$ by (a) above, for then $\sigma(S) \subset(W \otimes R) \cap(U \otimes B)=U \otimes(R \cap B)=U \otimes S$.

We now come to the situation we wish to study. Note that ${ }^{\operatorname{co} W} A \subset A$ is left $W$-Galois if the map $A \otimes_{\mathrm{coW}_{A}} A \rightarrow W \otimes A$ given by $x \otimes y \mapsto \sum x_{-1} \otimes x_{0} y$ is bijective; when $S_{W}$ is bijective this is equivalent to $x \otimes y \mapsto \sum x_{-1} \otimes y x_{0}$ being bijective. Thus $A$ is left $W$-Galois $\Longleftrightarrow A^{\text {cop }}$ is right $W^{\text {op cop }}$-Galois. The second map will be used later on.

2.6. Definition. Let $W$ and $H$ be Hopf algebras with bijective antipodes, $U \subset W$ a Hopf subalgebra such that $U \subset W$ is a faithfully flat extension of algebras, and $A$ a 
(W,H)-bicomodule algebra. As above, set $\bar{W}=W / W U^{+}, B={ }^{c o} \bar{W} A, R=A^{c o} H$, and $S=R \cap B$. Assume:

(a) $R \subset A$ and $S \subset B$ are faithfully flat $H$-Galois extensions, and

(b) ${ }^{\operatorname{co}} W A \subset A$ is a $W$-Galois extension.

Then $(U, W, H, A)$ is called a faithfully flat bi-Galois extension.

We represent this situation in the following commutative diagram:

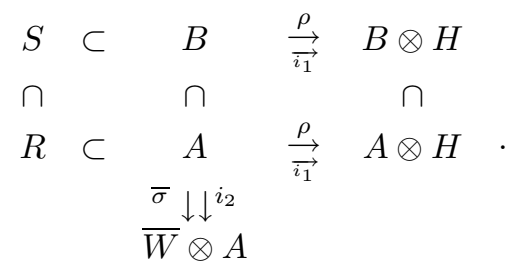

We note that bi-Galois extensions have also been studied recently by Schauenburg $[\mathrm{Sb}]$ in the following special case: $U=k 1$ (so $\bar{W}=W$ ) and $B=R=k$; that is, the coinvariants in $A$ for both $W$ and $H$ are trivial.

In all of our applications, we consider the following case:

2.7. Main Example. Let $H$ and $A$ be Hopf algebras with bijective antipodes and a surjective Hopf algebra map $\pi: A \rightarrow H$. Suppose $B$ is a Hopf subalgebra of $A$ such that $B \subset A$ is faithfully flat and such that $\pi: B \rightarrow H$ is also surjective. Consider $A$ as a left $A$-comodule via $\sigma=\triangle_{A}$ and as a right $H$-comodule via $\rho=(i d \otimes \pi) \circ \triangle_{A}$. Now set $R=A^{c o H}$ and $S=B^{c o H}$. Using $W=A$ and $U=B$, we have $\bar{W}=\bar{A}=A / A B^{+}$, and so $B={ }^{c o} \bar{A} A$ by Lemma 2.4 (a). Because of these simplifying assumptions, $(B, A, H, A)$ will be a faithfully flat bi-Galois extension provided $R \subset A$ and $S \subset B$ are faithfully flat $H$-Galois extensions.

We express this in the diagram

$$
\begin{array}{lllll}
S & \subset & B & \stackrel{\pi}{\rightarrow} & H \\
\cap & & \cap & & \| \\
R & \subset & A & \stackrel{\pi}{\rightarrow} & H
\end{array}
$$

One property which will guarantee that the $H$-Galois extensions $R \subset A$ and $S \subset B$ are faithfully flat is the existence of a total integral, that is, a right $H$ comodule map $\Gamma: H \rightarrow B$ such that $\Gamma(1)=1$; see $[\mathrm{KT}]$ or [Sch 90]. We consider two important special cases in which this property holds:

(a) The extension $S \subset B$ is cleft; that is, such a $\Gamma: H \rightarrow B$ exists which is (convolution) invertible. This is equivalent to assuming that $A$ and $B$ are crossed products; that is, $A=R \#_{\sigma} H$ and $B=S \#_{\sigma} H$, for some invertible cocycle $\sigma$ : $H \otimes H \rightarrow S$. In fact this will happen whenever $A$ is finite-dimensional or pointed, by [Sch 92]. Cleft extensions will be considered further in Section 4.

(b) Assume in addition that $\Gamma: H \rightarrow B$ is a Hopf algebra map such that $\pi \Gamma=i d$; that is, $B$ is a Hopf algebra with a projection. It now follows by Radford's theorem [R 85] that $B=S \star H$, a biproduct; similarly $A=R \star H$. The above diagram then becomes

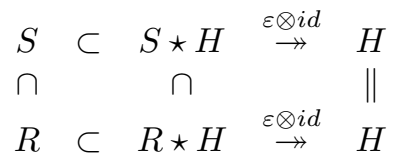


This is the situation discussed in detail in Section 5: that is, $S \subset R$ are Hopf algebras in the Yetter-Drinfeld category ${ }_{H}^{H} \mathcal{Y} \mathcal{D}$.

In fact the situation in (b) will always occur in the set-up of Example 2.7 whenever $S=k$. For, $R \subset A$ being $H$-Galois means that the Galois map $\gamma: A \otimes_{R} A \rightarrow$ $A \otimes H$ given by $a \otimes b \mapsto \sum a b_{1} \otimes \pi\left(b_{2}\right)$, for all $a, b \in A$, is bijective. Tensoring on the left by $k \cong A / A^{+}$as a right $A$-module, we see that $k \otimes_{R} A \cong H$, given by $1 \otimes b \mapsto \pi(b)$. Restricting this map to the $H$-Galois extension $k \subset B$, we see that $\pi$ restricted to $B$ is an isomorphism. Thus $B \cong H$, and there exists a Hopf algebra map $\Gamma: H \rightarrow B \subset A$ such that $\pi \Gamma=i d$.

We record some general facts about the situation of our main example.

2.8. Proposition. Let $(B, A, H, A)$ be a faithfully flat bi-Galois extension as in Example 2.7, with $R=A^{\text {co } H}$ and $S=B^{\text {co } H}$. Then:

(a) $R \otimes_{S} B \cong A$ via multiplication,

(b) $B \otimes_{S} R \cong A$ via multiplication,

(c) Let $\bar{R}=R / R S^{+}$and $\bar{A}=A / A B^{+}$. Then $\bar{R} \cong \bar{A}$, as left $R$-modules and right $S$-modules, via the map induced by the inclusion $R \subset A$.

Proof. (a) Let $\mathcal{M}_{B}^{H}$ be the category of right $(B, H)$-Hopf modules. Since $S \subset B$ is $H$-Galois and faithfully flat, by [Sch 90] there is a category equivalence $\mathcal{M}_{S} \leftrightarrows \mathcal{M}_{B}^{H}$, given by $M \mapsto M \otimes_{S} B$ and $V \mapsto V^{\text {co } H}$, for $M \in \mathcal{M}_{S}, V \in \mathcal{M}_{B}^{H}$. Now $A \in \mathcal{M}_{B}^{H}$ using the given $H$-comodule structure $\rho: A \rightarrow A \otimes H$, and $A^{\text {co } H}=R$; hence using $M=R$, the map $R \otimes_{S} B \rightarrow A$, given by $r \otimes b \mapsto r b$, is a bijection.

(b) This is similar to (a), using the equivalence ${ }_{S} \mathcal{M} \leftrightarrows_{B} \mathcal{M}^{H}$.

(c) First note that given any $S$-module $M$ and ideal $I$ of $S, M \otimes_{S} S / I \cong M / M I$. Thus using $I=S^{+}$, we have $M \otimes_{S} k \cong M / M S^{+}$. We apply this fact for $M=R$ and use $R \otimes_{S} B \cong A$ from part (a): $R / R S^{+} \cong R \otimes_{S} k \cong R \otimes_{S} B \otimes_{B} k \cong A \otimes_{B} k \cong$ $A / A B^{+}$.

\section{Frobenius extensions of SUbAlgebras}

In this section we prove our main result about when the coinvariants in a biGalois extension are $\beta$-Frobenius.

We recall the notion of bi-Galois extensions from Definition 2.6: $U \subset W$ and $H$ are Hopf algebras with bijective antipodes, $A$ is a $(W, H)$-bicomodule algebra, $\bar{W}=W / W U^{+}, R=A^{c o H}, B={ }^{c o} \bar{W} A$, and $S=R \cap B$, with various Galois and faithful flatness assumptions. The diagram in 2.6 may be helpful. Part (a) of the theorem is due to Schneider, as noted in Theorem 1.14.

3.1. Theorem. Let $(U, W, H, A)$ be a faithfully flat bi-Galois extension, and assume that $U \subset W$ is of right integral type, with $\lambda$ and $\chi$ as in Definition 1.10. Define

$$
\begin{aligned}
& f: A \rightarrow B \text { by } f(a):=\sum \lambda\left(\overline{a_{-1}}\right) a_{0} \\
& \beta: B \rightarrow B \text { by } \beta(b):=\sum \chi\left(b_{-1}\right) b_{0}
\end{aligned}
$$

for all $a \in A, b \in B$. Then: 
(a) $B \subset A$ is a $\beta$-Frobenius extension with Frobenius map $f$.

(b) $S \subset R$ is a $\beta_{R}$-Frobenius extension with Frobenius map $f_{R}$, where $f_{R}: R \rightarrow S$ and $\beta_{R}: S \rightarrow S$ are the restrictions of $f$ and $\beta$ to $R$ and $S$.

(c) We may choose dual bases as follows:

Let $\gamma: A \otimes_{\text {co }{ }_{A}} A \rightarrow W \otimes A$, via $x \otimes y \mapsto \sum y_{-1} \otimes x y_{0}$, be the Galois isomorphism, and let $r_{i}, l_{i} \in A, 1 \leq i \leq n$, be such that $\gamma\left(\sum r_{i} \otimes l_{i}\right)=\Lambda \otimes 1$. Then $\left\{r_{i}, l_{i}\right\}$ are dual bases of $B \subset A$.

Moreover, there exist $x_{j}, y_{j} \in R, 1 \leq j \leq m$, such that

$$
\sum_{j} x_{j} \otimes y_{j}=\sum_{i} r_{i} \otimes l_{i} \text { in } A_{\beta} \otimes_{B} A,
$$

and all elements $\left\{x_{j}, y_{j}\right\}$ having this property are dual bases of the $\beta_{R}$-Frobenius extension $S \subset R$ with respect to $f_{R}$ (and of the $\beta$-Frobenius extension $B \subset A$ ).

Proof. (a) This is Theorem 1.14, which was shown by constructing dual bases and using part (c) of Proposition 1.3.

(b) We make three preliminary observations.

(1) $f$ and $\beta$ restrict to $f_{R}$ and $\beta_{R}$ :

First, if $a \in R$, then $f(a)=\sum \lambda\left(\bar{a}_{-1}\right) a_{0} \in R$ since $\sigma(R) \subset W \otimes R$ by Lemma 2.3 (b), with $C=W, D=H$, and $E=R$. Similarly, if $b \in S$, then $\beta(b)=\sum \chi\left(b_{-1}\right) b_{0} \in$ $S$ since $\sigma(S) \subset U \otimes S$ by Lemma 2.5 (b).

Note that $\chi: U \rightarrow k$ is an algebra map by 1.10 (c), and hence both $\beta$ and $\beta_{R}$ are algebra automorphisms of $B$ and $S$, respectively, with inverse $b \mapsto \sum \chi\left(S b_{-1}\right) b_{0}$.

(2) $R_{S}$ is flat:

For $X \in{ }_{S} \mathcal{M}$, the functor

$$
X \mapsto A \otimes_{S} X \cong A \otimes_{B} B \otimes_{S} X \cong A \otimes_{R} R \otimes_{S} X
$$

is exact since $B_{S}$ is flat by the bi-Galois extension hypothesis and $A_{B}$ is flat since $B \subset A$ is $\beta$-Frobenius, by (a). Since also $R \subset A$ is faithfully flat by the bi-Galois hypothesis, it follows that $X \mapsto R \otimes_{S} X$ is exact.

(3) $R_{\beta_{R}} \otimes_{S} A \rightarrow A_{\beta} \otimes_{B} A$, via $r \otimes a \mapsto r \otimes a$, is bijective:

Note that

$$
\begin{aligned}
& R_{\beta_{R}} \otimes_{S} A \cong R \otimes_{S} \quad \beta_{R}^{-1} A, \text { via } r \otimes a \mapsto r \otimes a, \text { by } 1.2(\mathrm{~b}), \text { and } \\
& B_{\beta} \otimes_{B} A \cong \beta^{-1} A, \text { via } b \otimes a \mapsto \beta^{-1}(b) a .
\end{aligned}
$$

Thus

$$
R_{\beta_{R}} \otimes_{S} A \cong R \otimes_{S} \beta_{R}^{-1} A \cong R \otimes_{S} B_{\beta} \otimes_{B} A \cong A_{\beta} \otimes_{B} A,
$$

where the last isomorphism is Proposition 2.8 (a).

We now prove (b) by applying Proposition 1.3(b). Define $F$ and $\tilde{F}$ in terms of $f$ and $\beta$ and also $F_{R}, \tilde{F}_{R}$ in terms of $f_{R}$ and $\beta_{R}$; that is,

$$
\begin{aligned}
& F_{R}: R \rightarrow \operatorname{Hom}_{S}\left(R_{S}, S_{S}\right) \text { via } F_{R}(x)=\left(f_{R}\right) x, \text { and } \\
& \tilde{F}_{R}: R_{\beta_{R}} \otimes_{S} R \rightarrow \operatorname{Hom}_{S}\left(R_{S}, R_{S}\right) \text { via } \tilde{F}_{R}(x \otimes y)(a)=x f(y a) .
\end{aligned}
$$

By 1.3 and part (a) above, $F$ and $\tilde{F}$ are bijective, and we must show that $F_{R}$ and $\tilde{F}_{R}$ are bijective. 
To check that $F_{R}$ is bijective, we claim that the following diagram commutes:

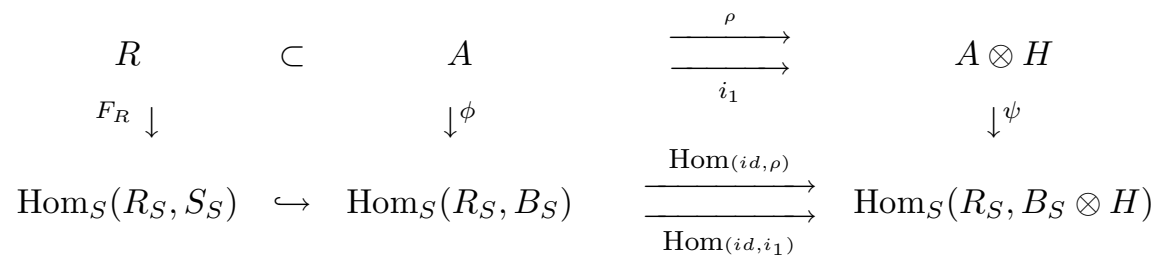

where $\phi(a)(r):=f(a r)$ and $\psi(a \otimes h)(r):=f(a r) \otimes h$. Clearly the left square commutes since both directions give $r \mapsto(x \mapsto f(r x))$, and similarly the right lower square commutes, with $a \mapsto(x \mapsto f(a x) \otimes 1)$. It remains to consider the upper right square. If $a \in A$, then $\psi \rho: a \mapsto \psi\left(\sum a_{0} \otimes a_{1}\right)=\left(x \mapsto \sum f\left(a_{0} x\right) \otimes a_{1}\right)$ and $\operatorname{Hom}(i d, \rho) \phi: a \mapsto(x \mapsto f(a x)) \mapsto(x \mapsto \rho(f(a x)))$. But now

$$
\begin{aligned}
\rho(f(a x)) & =\rho\left(\sum \lambda\left(\overline{a_{-1} x_{-1}}\right) a_{0} x_{0}\right) \\
& =\sum \lambda\left(\overline{a_{-1} x_{-1}}\right)\left(a_{0}\right)_{0}\left(x_{0}\right)_{0} \otimes\left(a_{0}\right)_{1}\left(x_{0}\right)_{1} \\
& =\sum \lambda\left(\overline{a_{-1} x_{-1}}\right)\left(a_{0}\right)_{0} x_{0} \otimes\left(a_{0}\right)_{1} \\
& =\sum \lambda\left(\overline{\left(a_{0}\right)_{-1} x_{-1}}\right)\left(a_{0}\right)_{0} x_{0} \otimes a_{1} \\
& =\sum f\left(a_{0} x\right) \otimes a_{1},
\end{aligned}
$$

where the third equality follows since $x \in R$ implies $x_{0} \in R$ (since $\sigma(R) \subset W \otimes R$ by Lemma $2.3(\mathrm{~b})$ ) and thus $\rho\left(x_{0}\right)=x_{0} \otimes 1$ since $R=A^{\text {co } H}$, and the fourth equality follows from the bicomodule condition. Thus the diagram commutes.

Now, the top row is exact since $R=A^{c o H}$; the bottom row is exact since $S=B^{\text {co } H}$ and since $\operatorname{Hom}_{S}\left(R_{S},-\right)$ is left exact.

$\phi$ is bijective, since $R \otimes_{S} B \rightarrow A$ is bijective by Proposition 2.8, and hence

$$
\phi: A \stackrel{F}{\rightarrow} \operatorname{Hom}_{B}\left(A_{B}, B_{B}\right) \cong \operatorname{Hom}_{B}\left(R \otimes_{S} B, B_{B}\right) \cong \operatorname{Hom}_{S}\left(R_{S}, B_{S}\right)
$$

is bijective since $F$ is bijective.

Finally, $\psi$ is bijective. For,

$\psi: A \otimes H \stackrel{F \otimes i d}{\longrightarrow} \operatorname{Hom}_{B}\left(A_{B}, B_{B}\right) \otimes H \stackrel{\cong}{\rightrightarrows} \operatorname{Hom}_{B}\left(A_{B}, B_{B} \otimes H\right) \cong \operatorname{Hom}_{S}\left(R_{S}, B_{S} \otimes H\right)$

where the last isomorphism follows from $R \otimes_{S} B \cong A$ as above and the middle mapping is given by $\varphi \otimes h \mapsto(a \mapsto \varphi(a) \otimes h)$; it is an isomorphism since $A_{B}$ is finitely-generated projective by part (a). Thus $\psi$ is bijective, and so $F_{R}$ is bijective using the diagram.

Now we show that $\tilde{F}_{R}$ is bijective. We claim that the following diagram commutes:

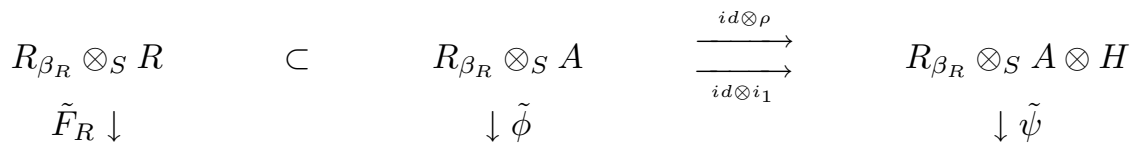

$$
\begin{aligned}
& \operatorname{Hom}_{S}\left(R_{S}, R_{S}\right) \stackrel{\operatorname{Hom}(i d, i)}{\longrightarrow} \operatorname{Hom}_{S}\left(R_{S}, A_{S}\right) \stackrel{\operatorname{Hom}(i d, \rho)}{\underset{\operatorname{Hom}\left(i d, i_{1}\right)}{\longrightarrow}} \operatorname{Hom}_{S}\left(R_{S}, A_{S} \otimes H\right)
\end{aligned}
$$

where $i: R \subset A$ is the inclusion map,

$$
\tilde{\phi}(x \otimes y)(r):=x f(y r), \text { and } \tilde{\psi}(x \otimes y \otimes h)(r):=x f(y r) \otimes h .
$$

The left square commutes since both possibilities give $x \otimes y \mapsto(r \mapsto x f(y r))$, and similarly the right lower square commutes, with $x \otimes y \mapsto(r \mapsto x f(y r) \otimes 1)$. It 
remains to consider the right upper square. For $x \in R_{\beta_{R}}, y \in A$, we have

$$
\tilde{\psi} \circ(i d \otimes \rho): x \otimes y \mapsto \tilde{\psi}\left(\sum x \otimes y_{0} \otimes y_{1}\right)=\left(r \mapsto \sum x f\left(y_{0} r\right) \otimes y_{1}\right) .
$$

The fact that $\operatorname{Hom}(i d, \rho) \circ \tilde{\phi}$ gives the same result follows using $\rho(f(y r))=$ $\sum f\left(y_{0} r\right) \otimes y_{1}$, which we showed above in the argument for $F_{R}$. Thus the diagram commutes.

The upper row is exact since, first, $R=A^{\text {co } H}$ implies $R \subset A \rightrightarrows A \otimes H$ is exact, and second, $R_{\beta_{R}}$ is flat as a right $S$-module (since $X_{\beta} \otimes_{S} Y \cong X \otimes_{S} \beta^{-1} Y$ for all $S$-modules $X$ and $Y$ by 1.2 (b), and hence $X$ flat as an $S$-module implies $X_{\beta}$ is also flat over $S$ ).

The lower row is also exact, since in fact $R \subset A \rightrightarrows A \otimes H$ is exact as $(R, R)$, and hence $(S, S)$-bimodules, and because $\operatorname{Hom}_{S}\left(R_{S},-\right)$ is left exact.

Next, $\tilde{\phi}$ and $\tilde{\psi}$ are bijective. $\tilde{\phi}$ is bijective since it is the composition of the isomorphisms

$$
\begin{aligned}
R_{\beta_{R}} \otimes_{S} A & \cong A_{\beta} \otimes_{B} A \text { by }(3) \\
& \stackrel{\tilde{F}}{\rightarrow} \operatorname{Hom}_{B}\left(A_{B}, A_{B}\right) \\
& \cong \operatorname{Hom}_{S}\left(R_{S}, A_{S}\right)
\end{aligned}
$$

where the last isomorphism is induced by $R \otimes_{S} B \cong A$, which is $2.8(\mathrm{a})$. $\tilde{\psi}$ is the composition of the isomorphisms

$$
\begin{aligned}
R_{\beta_{R}} \otimes_{S} A \otimes H & \cong A_{\beta} \otimes_{B} A \otimes H \quad \text { by }(3) \text { again } \\
& \stackrel{\vec{F} \otimes i d}{\longrightarrow} \operatorname{Hom}_{B}\left(A_{B}, A_{B}\right) \otimes H \\
& \cong \operatorname{Hom}_{B}\left(A_{B}, A_{B} \otimes H\right) \quad \text { since } A_{A} \text { is fin. gen. projective } \\
& \cong \operatorname{Hom}_{S}\left(R_{S}, A_{S} \otimes H\right) \quad \text { by 2.8(a) as above. }
\end{aligned}
$$

Using the diagram, it now follows that $\tilde{F}_{R}$ is bijective, proving (b).

(c) The following diagram commutes:

$$
\begin{aligned}
& R_{\beta_{R}} \otimes_{S} R \longrightarrow \tilde{F}_{R} \longrightarrow \operatorname{Hom}_{S}\left(R_{S}, R_{S}\right) \\
& g \downarrow \quad \downarrow_{\mathrm{Hom}(i d, i)} \\
& A_{\beta} \otimes_{B} A \stackrel{\tilde{F}}{\longrightarrow} \operatorname{Hom}_{B}\left(A_{B}, A_{B}\right) \cong \operatorname{Hom}_{S}\left(R_{S}, A_{S}\right)
\end{aligned}
$$

where the lower right isomorphism is induced by $R \otimes_{S} B \cong A$ and the map $g$ is the isomorphism of (b) (3) restricted to $R_{\beta_{R}} \otimes_{S} R$.

Now by Proposition 1.3 and the proof there of $(b) \Rightarrow(c)$, it follows that if $B \subset A$ is a $\beta$-Frobenius extension with Frobenius homomorphism $f$, and $\tilde{F}$ is as above, then $\left\{r_{i}, l_{i}\right\}$ are dual bases $\Leftrightarrow \tilde{F}\left(\sum_{i} r_{i} \otimes l_{i}\right)=i d_{A} \Leftrightarrow \sum_{i} r_{i} f\left(l_{i} x\right)=x$, for all $x \in A$, where we consider $\sum r_{i} \otimes l_{i} \in A_{\beta} \otimes_{B} A$.

It follows that if $\left\{r_{i}, l_{i}\right\}$ are chosen in $A$ such that $\gamma\left(\sum r_{i} \otimes l_{i}\right)=\Lambda \otimes 1$, then $\left\{r_{i}, l_{i}\right\}$ are dual bases of $B \subset A$. Indeed, for all $x \in A$,

$$
\begin{aligned}
\sum r_{i} f\left(l_{i} x\right) & =\sum r_{i} \lambda\left(\overline{\left(l_{i}\right)_{-1} x_{-1}}\right)\left(l_{i}\right)_{0} x_{0} \\
& =\sum \lambda\left(\overline{\Lambda x_{-1}}\right) x_{0} \quad \text { since } \sum\left(l_{i}\right)_{-1 \beta} \otimes r_{i}\left(l_{i}\right)_{0}=\Lambda \otimes 1 \\
& =\sum \varepsilon\left(x_{-1}\right) x_{0}=x .
\end{aligned}
$$


Now by (b), since $S \subset R$ is $\beta$-Frobenius, there exist dual bases $\left\{x_{j}, y_{j}\right\}$ of $S \subset R$; thus $\tilde{F}_{R}\left(\sum x_{j} \otimes_{S} y_{j}\right)=i d_{R}$ by the above. By commutativity of the diagram, it must be that $g\left(\sum x_{j} \otimes_{S} y_{j}\right)=\sum x_{j} \otimes_{B} y_{j}=\sum r_{i} \otimes l_{i}$ in $A_{\beta} \otimes_{B} A$.

Finally, given $\left\{r_{i}, l_{i}\right\}$ dual bases for $B \subset A$, assume that $\left\{x_{j}, y_{j}\right\}$ are elements of $R$ such that $\sum x_{j} \otimes y_{j}=\sum r_{i} \otimes l_{i}$ in $A_{\beta} \otimes_{B} A$. Then

$$
\tilde{F}\left(\sum x_{j} \otimes_{B} y_{j}\right)=\tilde{F}\left(\sum r_{i} \otimes l_{i}\right)=i d_{A} .
$$

Since the above diagram commutes, it follows that $\operatorname{Hom}(i d, i) \tilde{F}_{R}\left(\sum x_{j} \otimes_{S} y_{j}\right)=i$ and thus that $\tilde{F}_{R}\left(\sum x_{j} \otimes_{S} y_{j}\right)=i d_{R}$, since $\operatorname{Hom}(i d, i)$ is injective and $\operatorname{Hom}(i d, i)\left(i d_{R}\right)$ $=i$. Thus $\left\{x_{j}, y_{j}\right\}$ are dual bases of $S \subset R$.

3.2. Remark. In the theorem, $\beta^{-1}$ can be described as the restriction of a function which is defined on $A$ and not just on $B$. For

$$
\begin{aligned}
& \beta^{-1}(a):=\sum \lambda\left(\overline{S\left(a_{-1}\right) \Lambda}\right) a_{0}, \quad \text { for all } a \in A, \\
& \beta^{-1}(a b)=\beta^{-1}(a) \beta^{-1}(b), \quad \text { for all } a \in A, b \in B .
\end{aligned}
$$

Consequently $\sum_{j} \beta^{-1}\left(x_{j}\right) y_{j}=\varepsilon(\Lambda)$, where $\left\{x_{j}, y_{j}\right\}$ are as in Theorem 3.1(c).

Proof. (a) First note that for $b \in B, \beta^{-1}(b)=\sum \chi\left(\overline{S b_{-1}}\right) \lambda(\bar{\Lambda}) b_{0}=\sum \chi\left(\overline{S b_{-1}}\right) b_{0}$ using the form of $\beta$ in Theorem 3.1 (and 1.14) and the fact that $\lambda(\bar{\Lambda})=1$, as noted after Definition 1.10. Consequently $\beta^{-1}(\beta(b))=b$ since $\chi$ is multiplicative. Now for all $a \in A, b \in B$,

$$
\begin{aligned}
\beta^{-1}(a b) & =\sum \lambda\left(S\left(a_{1} b_{-1}\right) \bar{\Lambda}\right) a_{0} b_{0} \\
& =\sum_{-1} \chi\left(S b_{-1}\right) \lambda\left(S a_{-1} \bar{\Lambda}\right) a_{0} b_{0} \quad \text { by } 1.10(\mathrm{c}) \\
& =\beta^{-1}(a) \sum \chi\left(S b_{-1}\right) b_{0} \\
& =\beta^{-1}(a) \beta^{-1}(b) .
\end{aligned}
$$

(b) Now by (a), the map $A_{\beta} \otimes_{B} A \rightarrow A$, via $x \otimes y \mapsto \beta^{-1}(x) y$, is well-defined, and thus $\sum_{j} \beta^{-1}\left(x_{j}\right) y_{j}=\sum_{i} \beta^{-1}\left(r_{i}\right) l_{i}$.

Recall we have chosen $\left\{r_{i}, l_{i}\right\}$ so that $\gamma\left(\sum r_{i} \otimes l_{i}\right)=\sum\left(l_{i}\right)_{-1} \otimes r_{i}\left(l_{i}\right)_{0}=\Lambda \otimes 1$. Under the map $W \otimes A \rightarrow W \otimes A$, via $w \otimes a \mapsto \sum a_{-1} \bar{S} w \otimes a_{0}$, we see that $\Lambda \otimes 1 \mapsto \bar{S} \Lambda \otimes 1$. It follows that

$$
\gamma\left(\sum l_{i} \otimes r_{i}\right)=\sum\left(r_{i}\right)_{-1} \otimes\left(r_{i}\right)_{0} l_{i}=\bar{S} \Lambda \otimes 1 .
$$

Now

$$
\begin{aligned}
\sum \beta^{-1}\left(r_{i}\right) l_{i} & =\sum \lambda\left(S\left(\left(r_{i}\right)_{-1}\right) \bar{\Lambda}\right)\left(r_{i}\right)_{0} l_{i} \\
& =\lambda(S(\bar{S} \Lambda) \bar{\Lambda}) \\
& =\lambda(\Lambda \cdot \bar{\Lambda})=\varepsilon(\Lambda) \quad \text { by } 1.10(\mathrm{~b}) .
\end{aligned}
$$

We consider the case of our main example, 2.7 , and show that more can be said. The reader should compare this result with Theorem 1.7.

3.3. Corollary. Assume we are in the situation of Example 2.7, that is, $B \subset A$ and $H$ are Hopf algebras, $B \subset A$ is a faithfully flat extension, $\pi: A \rightarrow H$ is a Hopf surjection which is also surjective when restricted to $B$. Let $R=A^{c o H}$ and $S=B^{c o H}$ using the $H$-comodule structure induced on $A$ and $B$ by $\pi$. Assume that $R \subset A$ and $S \subset B$ are faithfully flat $H$-Galois extensions, and that $B \subset A$ is of right integral type. Then $S \subset R$ is $\beta$-Frobenius, with $\beta$ and $f$ as in Theorem 3.1, and: 
(a) The element $\Lambda \in A(=W)$ in 1.10 (b) can be chosen in $R$.

(b) Dual bases of $S \subset R$ may be found as follows:

There exist $x_{i}, y_{i} \in R, 1 \leq i \leq m$, such that in $A_{\beta} \otimes_{B} A$,

$$
\sum x_{i} \otimes y_{i}=\sum \bar{S} \Lambda_{2} \otimes \Lambda_{1}
$$

More generally, for any $r \in R$, there exist $x_{i}, y_{i} \in R$ such that in $A_{\beta} \otimes_{B} A$,

$$
\sum x_{i} \otimes y_{i}=\sum \bar{S} r_{2} \otimes r_{1} .
$$

In particular $\left\{\bar{S} \Lambda_{2}, \Lambda_{1}\right\}$ are dual bases for $B \subset A$, and $\left\{x_{i}, y_{i}\right\}$ are dual bases for $S \subset R$.

Proof. Using $W=A$ and $U=B$, we see that the assumptions imply that ( $B, A, H, A)$ is a faithfully flat bi-Galois extension. Thus Theorem 3.1 applies.

(a) By 2.8 (b), $A=B R$; hence there exist $b_{i} \in B, u_{i} \in R, 1 \leq i \leq n$, such that $\Lambda=\sum_{i} b_{i} u_{i}$. Then, using $1.10(\mathrm{~b})$ and $(\mathrm{c})$,

$$
\varepsilon=\lambda \cdot \Lambda=\sum_{i}\left(\lambda \cdot b_{i}\right) u_{i}=\sum_{i} \chi\left(b_{i}\right) \lambda \cdot u_{i}=\lambda \cdot \sum_{i} \chi\left(b_{i}\right) u_{i}=\lambda \cdot \Lambda^{\prime},
$$

where $\Lambda^{\prime}=\sum_{i} \chi\left(b_{i}\right) u_{i} \in R$.

(b) Given $r \in R$, and any $z \in R$,

$$
\begin{aligned}
\tilde{F}\left(\sum \bar{S} r_{2} \otimes r_{1}\right)(z) & =\sum\left(\bar{S} r_{2}\right) f\left(r_{1} z\right) \\
& =\sum \bar{S} r_{3} \lambda\left(\overline{r_{1} z_{1}}\right) r_{2} z_{2} \quad \text { by the definition of } f \\
& =\sum\left(\bar{S} r_{3}\right) r_{2} \lambda\left(\overline{r_{1} z_{1}}\right) z_{2} \\
& =\sum \lambda\left(\bar{r} z_{1}\right) z_{2} \in R .
\end{aligned}
$$

Thus $\tilde{F}\left(\sum \bar{S} r_{2} \otimes r_{1}\right) \in \operatorname{Hom}_{S}(R, R)$.

By the diagram used in the proof of Theorem 3.1 (c), it follows that $\sum \bar{S} r_{2} \otimes r_{1}$ lies in the image of $R_{\beta_{R}} \otimes S R$ under $g$; that is, $\sum \bar{S} r_{2} \otimes r_{1}=\sum x_{i} \otimes y_{i}$, for some $x_{i}, y_{i} \in R$.

The first statement now follows, choosing $\Lambda \in R$ as in (a), or using Theorem 3.1(c) since under the Galois map $\gamma: A \otimes A \rightarrow A \otimes A$ given by $x \otimes y \mapsto$ $\sum y_{1} \otimes x y_{2}$, we have $\gamma\left(\sum \bar{S} \Lambda_{2} \otimes \Lambda_{1}\right)=\Lambda \otimes 1$.

\section{EXtensions of InTEGRAL TYPE}

It is clear from Theorem 1.14 (from [Sch 92]) and from our Theorem 3.1 that it is important to find conditions which guarantee that an extension of Hopf algebras is of integral type. We do this here by generalizing the methods of Larson and Sweedler [LS] to cleft extensions of finite index. In fact we consider a more general situation than that of Hopf subalgebras; for most of what we do, a right coideal subalgebra will suffice.

As in Section 2, let $K$ be a right coideal subalgebra of the Hopf algebra $H$ and let $\bar{H}=H / H K^{+} ; \bar{H}$ is a coalgebra and a left $H$-module.

4.1. Definition. Let $K \subset H$ be a right coideal subalgebra of $H$ and let $\bar{H}=$ $H / H K^{+}$. Then $H$ has a right normal basis over $K$ if $H \cong \bar{H} \otimes K$ as right $K$ modules and left $\bar{H}$-comodules. Here $H$ has the induced left $\bar{H}$-comodule structure. 
4.2. Remark. If $H$ has a right normal basis over $K$, then $H$ is free over $K$, and so $K \subset H$ is right faithfully flat. Thus, by Lemma 2.4 we know that $K={ }^{c o} \bar{H} H$ and that the Galois map $\gamma: H \otimes_{K} H \rightarrow \bar{H} \otimes H$, via $x \otimes y \mapsto \sum \overline{x_{1}} \otimes x_{2} y$, is a bijection.

In addition, the Galois map $\gamma$ is also a bijection, when applied to $H \otimes_{k} K$ :

$$
\gamma: H \otimes K \rightarrow H \square \bar{H} H .
$$

This last fact is dual to Lemma 2.4(a) and requires $K={ }^{c o} \bar{H} H$.

Similarly, one could define a normal basis property given a quotient left module and coalgebra of $H$. We next require a known lemma [T 79, Theorem 1], although we sketch a direct proof for completeness.

4.3. Lemma. Let $\bar{H}=H / I$ be a quotient coalgebra and left $H$-module. Define $K:={ }^{c o}{ }^{\bar{H}} H$, a right coideal subalgebra. Assume $H \cong \bar{H} \otimes K$ as right $K$-modules and left $\bar{H}$-comodules. Then $I=H K^{+}$, and so $H$ has a right normal basis over $K$ as in Definition 4.1.

Proof. This is dual to Lemma 2.4(b), since $H$ is left $\bar{H}$-faithfully coflat. For, consider the diagram

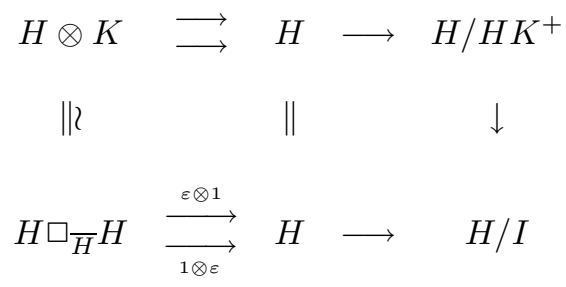

Note that $\mathrm{HK}^{+} \subset I$, and hence the diagram is commutative.

4.4. Remark. In the situation of Definition 4.1, the following are all well-defined categories of Hopf modules:

$$
{ }_{H}^{\bar{H}} \mathcal{M},{ }_{H} \mathcal{M}^{\bar{H}}, \mathcal{M}_{K}^{H},{ }_{K} \mathcal{M}^{H} .
$$

For, a $k$-space $M$ is in ${ }_{H}^{\bar{H}} \mathcal{M}$ if it is a left $H$-module, a left $\bar{H}$-comodule via $\rho$ : $M \rightarrow \bar{H} \otimes M$, and $\rho$ is a left $H$-module map. This is well-defined since $\bar{H}$ is a left $H$-module; similarly for ${ }_{H} \mathcal{M}^{\bar{H}}$. The second two are well-defined because $K$ is a right coideal subalgebra.

We require some known facts about Hopf modules.

4.5. Proposition ([Sch 90], [MD]). Assume that $H$ is $\bar{H}$-cleft, with $K={ }^{c o}{ }^{\bar{H}} H$. Then the following are inverse equivalences:

(a)

$$
\begin{array}{ccc}
{ }_{K} \mathcal{M} & \leftrightarrows & { }_{H}^{\bar{H}} \mathcal{M}, \\
M & \mapsto & H \otimes_{K} M, \\
{ }^{c o} \bar{H} V & \hookrightarrow & V .
\end{array}
$$

In particular, for any $V \in \in_{H}^{\bar{H}} \mathcal{M}, H \otimes{ }_{K}^{c o} \bar{H} V \dot{\rightarrow}$ is an isomorphism, where $\cdot$ is the action of $H$ on $V$. 
(b)

$$
\begin{array}{ccc}
\mathcal{M}^{\bar{H}} & \leftrightarrows & \mathcal{M}_{K}^{H}, \\
M & \mapsto & M \square_{\bar{H}} H, \\
\bar{V}:=V / V K^{+} & \longleftrightarrow & V .
\end{array}
$$

In particular, for any $V \in \mathcal{M}_{K}^{H}, V \rightarrow \bar{V} \square_{\bar{H}} H$ is an isomorphism, given by the comodule structure of $V$.

Proof. (a) is a special case of [Sch 90,3.7], since $K \subset H$ is faithfully flat, and (b) is a special case of $[\operatorname{Sch} 90,4.7]$.

(a) and (b) also follow from [MD] using $\gamma$ and its dual in 4.2.

We consider various structures on duals of (co)modules.

4.6. Definition. (a) Transposed (co)actions: If $A$ is an algebra and $V$ a left $A$-module, then $V^{*}=\operatorname{Hom}(V, k)$ becomes a right $A$-module via $(f \cdot a)(v):=f(a v)$, for all $v \in V . V^{*}$ is called the transposed $A$-module; similarly for right $A$-modules.

Note that if $A$ acts on $V=A$ by left multiplication, then the transposed action of $A$ on $A^{*}$ is simply $f \cdot a=f \leftarrow a$, as discussed in Section 0 .

Dually, let $C$ be a coalgebra and $V \rightarrow V \otimes C, v \mapsto \sum v_{0} \otimes v_{1}$, a finite-dimensional right $C$-module. Then $V^{*}$ is a left $C$-comodule via $V^{*} \rightarrow C \otimes V^{*}, f \mapsto \sum f_{-1} \otimes f_{0}$, where for all $v \in V$,

$$
\sum f_{0}(v) f_{-1}:=\sum f\left(v_{0}\right) v_{1}
$$

$V^{*}$ is called the transposed $C$-comodule. Similarly we may begin with a finitedimensional left $C$-comodule.

(b) Contragredient (co)actions: Let $H$ be a Hopf algebra. If $V$ is a left $H$-module, then the contragredient left $H$-module structure on $V^{*}$ is defined by $(h \cdot f)(v):=f((S h) \cdot v)$, for all $v \in V$. Similarly we may begin with a right $H$-module.

Note that if $H$ acts on $V=H$ by left multiplication, then the contragredient left $H$-action on $H^{*}$ is $h \cdot f=f \leftarrow S h$.

If $V$ is a finite-dimensional right $H$-comodule, then the contragredient right $H$ comodule structure on $V^{*}$ is defined by $f \mapsto \sum f_{0} \otimes S f_{-1}$, where $f \mapsto \sum f_{-1} \otimes f_{0}$ is the transposed left comodule structure above. Similarly we may begin with a finite-dimensional left $H$-comodule.

4.7. Lemma. Let $H$ be a Hopf algebra, $K \subset H$ a right coideal subalgebra, and $\bar{H}=H / I$ a left $H$-module coalgebra quotient.

(a) Let $V \in{ }_{H} \mathcal{M}^{\bar{H}}$ with $\operatorname{dim} V<\infty$. Then $V^{*} \in{ }_{H}^{\bar{H}} \mathcal{M}$, where $V^{*}$ is the transposed $\bar{H}$-comodule and the contragredient $H$-module.

(b) Let $V \in{ }_{K} \mathcal{M}^{H}$ with $\operatorname{dim} V<\infty$. Then $V^{*} \in \mathcal{M}_{K}^{H}$, where $V^{*}$ is the contragredient $H$-comodule and the transposed $K$-module.

Proof. (a) This is similar to the Larson-Sweedler argument [LS]. Let $\delta_{V}: V \rightarrow$ $V \otimes \bar{H}, v \mapsto \sum v_{0} \otimes v_{1}$, and $\delta_{V^{*}}: V^{*} \rightarrow \bar{H} \otimes V^{*}$, via $f \mapsto \sum f_{-1} \otimes f_{0}$, be the $\bar{H}$-comodule structure and its transpose, as in 4.6. We must show $\delta_{V^{*}}(h \cdot f)=$ $\sum h_{1} f_{-1} \otimes h_{2} \cdot f_{0}$, for all $f \in V^{*}, h \in H$, where $h \cdot f$ is the contragredient $H$-module action. That is, for all $v \in V$, we need

$$
\sum\left(h_{2} \cdot f_{0}\right)(v)\left(h_{1} \cdot f_{-1}\right)=\sum(h \cdot f)\left(v_{0}\right) v_{1}
$$


or equivalently $\sum f_{0}\left(\left(S h_{2}\right) \cdot v\right)\left(h_{1} \cdot f_{-1}\right)=\sum f\left(S h \cdot v_{0}\right) v_{1}$. Now

$$
\begin{aligned}
\sum f_{0}\left(\left(S h_{2}\right) \cdot v\right)\left(h_{1} \cdot f_{-1}\right) & \left.=\sum h_{1} \cdot\left(f\left(\left(S h_{2}\right) \cdot v\right)_{0}\right)\left(S h_{2} \cdot v\right)_{1}\right) \quad \text { by } 4.6(\mathrm{a}) \\
& =\sum h_{1} \cdot\left(f\left(\left(S h_{3}\right) \cdot v_{0}\right)\left(S h_{2} \cdot v_{1}\right)\right) \quad \text { since } V \in{ }_{H} \mathcal{M}^{\bar{H}} \\
& =\sum f\left((S h) \cdot v_{0}\right) v_{1} .
\end{aligned}
$$

(b) This is dual to (a). Let $\triangle_{V}: V \rightarrow V \otimes H, v \mapsto \sum v_{0} \otimes v_{1}$, and $V^{*} \rightarrow$ $H \otimes V^{*}, f \mapsto \sum f_{-1} \otimes f_{0}$, be the $H$-comodule structure and its transpose. Then $\triangle_{V^{*}}: V^{*} \rightarrow V^{*} \otimes H, f \mapsto \sum f_{0} \otimes S f_{-1}$, is the $H$-comodule structure of $V^{*} \in \mathcal{M}_{K}^{H}$.

We must show that $\triangle_{V^{*}}(f \cdot k)=\sum f_{0} \cdot k_{1} \otimes\left(S f_{-1}\right) k_{2}$, for all $k \in K, f \in V^{*}$; that is, for all $v \in V$,

$$
\sum\left(f_{0} \cdot k_{1}\right)(v)\left(S f_{-1}\right) k_{2}=\sum(f \cdot k)\left(v_{0}\right) S v_{1},
$$

or equivalently

Now

$$
\sum f_{0}\left(k_{1} \cdot v\right)\left(S f_{-1}\right) k_{2}=\sum f\left(k \cdot v_{0}\right) S v_{1}
$$

$$
\begin{aligned}
\sum f_{0}\left(k_{1} \cdot v\right)\left(S f_{-1}\right) k_{2} & =\sum f\left(\left(k_{1} \cdot v\right)_{0}\right) S\left(\left(k_{1} \cdot v\right)_{1}\right) k_{2} \quad \text { by } 4.6(1) \\
& =\sum f\left(k_{1} \cdot v_{0}\right) S\left(k_{2} \cdot v_{1}\right) k_{3} \quad \text { since } V \in{ }_{K} \mathcal{M}^{H} \\
& =\sum f\left(k \cdot v_{0}\right) S v_{1} .
\end{aligned}
$$

We return now to the situation of Definition 4.1. That is, $K$ is a right coideal subalgebra of the Hopf algebra $H$, and $\bar{H}=H / H K^{+}$. Then $\bar{H}^{*}$ is an algebra, since $\bar{H}$ is a coalgebra; moreover $\bar{H}^{*}$ is augmented via $f \mapsto f(\overline{1})$, and thus we may discuss the existence of integrals in $\bar{H}^{*}$. Note also that $\bar{H}^{*}$ is a right $H$-module, considered as the transposed right $H$-module of $\bar{H}$ considered as a left $H$-module. That is, given $f \in \bar{H}^{*}, h \in H$, and $\bar{g} \in \bar{H}$, we have $(f \cdot h)(\bar{g})=f(\overline{h g})$.

The following theorem lays the foundation to give cases of extensions that are of (right) integral type.

4.8. Theorem. Let $H$ be a Hopf algebra, $K$ a right coideal subalgebra, and $\bar{H}=$ $H / H K^{+}$as above. Assume $H$ has a right normal basis over $K$, and that $\operatorname{dim} \bar{H}<$ $\infty$. Then:

(a) $\int_{\bar{H}^{*}}^{r}$ is one-dimensional, with basis $\lambda \neq 0$.

(b) There exists $\Lambda \in H$ such that $\lambda \leftarrow \Lambda=\varepsilon$ on $\bar{H}$.

(c) There exists $\tilde{\chi} \in \operatorname{Alg}(K, k)$ such that $\lambda \leftarrow S k=\tilde{\chi}(k) \lambda$ for all $k \in K$.

(d) $\bar{H}^{*}$ is a Frobenius algebra.

Proof. First, $\bar{H} \in{ }_{H} \mathcal{M}^{\bar{H}}$, where the right $\bar{H}$-comodule structure is given by $\triangle_{\bar{H}}$, that is, $\bar{H} \rightarrow \bar{H} \otimes \bar{H}$ via $\bar{h} \mapsto \sum \bar{h}_{1} \otimes \bar{h}_{2}$, and $\bar{H}$ is a left $H$-module as usual. Thus $\bar{H}^{*} \in{ }_{H}^{\bar{H}} \mathcal{M}$ by Lemma $4.7(\mathrm{a})$, and so, by Proposition 4.5,

$$
H \otimes_{K}{ }^{\text {co }} \bar{H}^{*} \bar{H}^{*} \longrightarrow \bar{H}^{*}
$$

is an isomorphism. $\bar{H}^{*}$ is the transposed $\bar{H}$-comodule; hence

$$
\begin{aligned}
{ }^{c o} \bar{H} \bar{H}^{*} & =\left\{\varphi \in \bar{H}^{*} \mid \forall \bar{h} \in \bar{H}, \sum \varphi_{0}(\bar{h}) \varphi_{-1}=\varphi(\bar{h}) \overline{1}\right\} \\
& \left.=\left\{\varphi \in \bar{H}^{*} \mid \forall \bar{h} \in \bar{H}, \varphi(\bar{h}) \overline{1}=\sum \varphi\left(\bar{h}_{1}\right) \bar{h}_{2}\right)\right\} \quad \text { by } 4.6(1) \\
& =\int_{\bar{H}^{*}}^{r},
\end{aligned}
$$


where the last equality follows as noted in Section 0 .

Also $H \cong \bar{H} \otimes K$ as right $K$-modules and left $\bar{H}$-comodules by assumption. Thus using $(*)$, we obtain

$$
\bar{H}^{*} \cong H \otimes_{K} \int_{\bar{H}^{*}}^{r} \cong(\bar{H} \otimes K) \otimes_{K} \int_{\bar{H}^{*}}^{r} \cong \bar{H} \otimes \int_{\bar{H}^{*}}^{r},
$$

where the isomorphism is as left $\bar{H}$-comodules. The result now follows:

(a) Clearly $\int_{\bar{H}^{*}}^{r}$ is one-dimensional (hence in particular non-zero), since $\operatorname{dim} \bar{H}=$ $\operatorname{dim} \bar{H}^{*}<\infty$. Pick $0 \neq \lambda \in \int_{\bar{H}^{*}}^{r}$

(b) Since $H \otimes_{K} \int_{\bar{H}^{*}}^{r} \longrightarrow \bar{H}^{*}$ is surjective, given by $h \otimes \lambda \mapsto h \cdot \lambda=\lambda \leftarrow S h$, it follows that there exists $\tilde{\Lambda} \in H$ such that $\lambda \leftarrow S \tilde{\Lambda}=\varepsilon$ on $\bar{H}$. Pick $\Lambda=S \tilde{\Lambda}$; then $\lambda \leftarrow \Lambda=\varepsilon$ on $\bar{H}$.

(c) Since $\int_{\bar{H}^{*}}^{r}={ }^{c o} \bar{H} \bar{H}^{*} \in{ }_{K} \mathcal{M}$, it follows that $\int_{\bar{H}^{*}}^{r} \leftarrow S K=\int_{\bar{H}^{*}}^{r}$. and so there exists $\tilde{\chi} \in K^{*}$ such that $\lambda \leftarrow S l=\tilde{\chi}(l) \lambda \quad \forall l \in K$. Note that by its definition, $\tilde{\chi}$ must be in $\operatorname{Alg}(K, k)$.

(d) First, we see that $(* *)$ determines an isomorphism $\phi: \bar{H} \rightarrow \bar{H}^{*}$ of left $\bar{H}$-comodules.

Taking transposes, $\phi^{*}:\left(\bar{H}^{*}\right)^{*} \rightarrow \bar{H}^{*}$ is an isomorphism of left $\bar{H}^{*}$-modules (for since $C=\bar{H}$ is finite-dimensional, any finite-dimensional left $C$-comodule $V$ dualizes to a left $C^{*}$-module; that is, $V \rightarrow C \otimes V$ gives $\left.C^{*} \otimes V^{*} \rightarrow V^{*}\right)$. Now since $\bar{H}^{*} \cong \bar{H}^{* *}$ as $\bar{H}^{*}$-modules, $\bar{H}^{*}$ is Frobenius.

It may be useful to describe these isomorphisms more explicitly. Let $\lambda$ be a basis of $\int_{\bar{H}^{*}}^{r}$. For $h \in H$, the action of $h$ on $\lambda$ in the isomorphism $(*)$ is given by $h \cdot \lambda=\lambda \longleftarrow S h$. In $(* *)$ we have identified $\bar{H}$ as a subspace of $H$ via the cleft map. That is, let $\gamma: \bar{H} \rightarrow \bar{H} \otimes K \cong H$ via $\bar{h} \mapsto \bar{h} \otimes 1$. Then $\phi$ is given by $\bar{h} \mapsto \lambda \leftarrow(S \gamma(\bar{h}))$.

Now consider the isomorphism $\phi^{*}: \bar{H}^{* *} \rightarrow \bar{H}^{*}$, where $\bar{H}^{*}$ is a left $\bar{H}^{*}$-module by left multiplication. The left $\bar{H}^{*}$-module structure of $\bar{H}^{* *}$ is transposed to the right $\bar{H}^{*}$-module structure defined by multiplication on $\bar{H}^{*}$ as noted above; explicitly, the transposed $\bar{H}$-comodule structure $\bar{H}^{*} \rightarrow \bar{H} \otimes \bar{H}^{*}, g \mapsto \sum g_{-1} \otimes g_{0}$, is defined by $\sum g_{0}(\bar{h}) g_{-1}=\sum g\left(\bar{h}_{1}\right) \bar{h}_{2}$, for all $\bar{h} \in \bar{H}$. Thus, the dual $\bar{H}^{*}$-module structure on $\bar{H}^{* *}$ is given by

$$
\begin{aligned}
& \bar{H}^{*} \otimes \bar{H}^{* *} \rightarrow \bar{H}^{* *}, \\
& f \otimes \varphi \quad \mapsto \quad\left(g \mapsto \sum f\left(g_{-1}\right) \varphi\left(g_{0}\right)\right) .
\end{aligned}
$$

This action is in fact $f \rightarrow \varphi$. For, note that for all $\bar{h} \in \bar{H}, \sum f\left(g_{-1}\right) g_{0}(\bar{h})=$ $\sum g\left(\bar{h}_{1}\right) f\left(\bar{h}_{2}\right)=g f(\bar{h})$, and so $\sum f\left(g_{-1}\right) g_{0}=g f$. Thus

$$
\begin{aligned}
(f \cdot \varphi)(g) & :=\sum f\left(g_{-1}\right) \varphi\left(g_{0}\right)=\varphi\left(\sum f\left(g_{-1}\right) g_{0}\right) \\
& =\varphi(g f)=(f \rightarrow \varphi)(g),
\end{aligned}
$$

which is what we would expect.

Part (b) of the next result has already been shown in Example 1.12. Part (c) extends work of [Sch 92], where it was shown that when $H$ is pointed then $K \subset H$ is $\bar{H}$-cleft.

4.9. Corollary. Let $H$ be a Hopf algebra, $K \subset H$ a Hopf subalgebra, and $\bar{H}=$ $\mathrm{H} / \mathrm{HK}^{+}$as above. Assume that $\bar{H}$ is finite dimensional, and that either 
(a) $H$ has a right normal basis over $K$ and the antipode of $K$ is bijective, or

(b) $H$ is finite-dimensional, or

(c) the coradical of $H$ is cocommutative.

Then $K \subset H$ is of right integral type and is a $\beta$-Frobenius extension. Moreover, $\bar{H}^{*}$ is a Frobenius algebra.

Proof. (a) Since $H$ has a right normal basis over $K$, Theorem 4.8 applies. Noting that $K$ is a Hopf subalgebra, we see that $S K \subset K$, and so we may take $\chi=\tilde{\chi} \circ \bar{S}$ to get 1.5 (c). Thus $K \subset H$ is of right integral type.

(b) This is a special case of (a), since by [Sch 92, 2.4(2b)], $K \subset H$ is $\bar{H}$-cleft whenever $H$ is finite-dimensional. But cleftness implies the normal basis isomorphism.

For case (c), let $\bar{k}$ denote the algebraic closure of our base field $k$ and consider $K^{\prime}=\bar{k} \otimes K \subset H^{\prime}=\bar{k} \otimes H$. Now $H^{\prime}$ is pointed, and thus $K^{\prime} \subset H^{\prime}$ is $\overline{H^{\prime}}$-cleft by [Sch 92, 4.3(2)]. Thus Theorem 4.8 applies to give that $K^{\prime} \subset H^{\prime}$ is of right integral type, and that ${\overline{H^{\prime}}}^{*}$ is a Frobenius algebra.

However $\overline{H^{\prime}}=H^{\prime} / H^{\prime}\left(K^{\prime}\right)^{+} \cong \bar{k} \otimes \bar{H}$ as coalgebras, and so $\bar{k} \otimes \bar{H}^{*} \cong{\overline{H^{\prime}}}^{*}$ as algebras. Thus $\bar{H}^{*}$ is Frobenius, and $\int_{\bar{H}^{*}}^{r}$ is one-dimensional. The other properties also clearly descend from $K^{\prime} \subset H^{\prime}$ to $K \subset H$. Thus $K \subset H$ is of right integral type.

Finally, when $H_{0}$ is cocommutative $K \subset H$ is faithfully flat by [T 72]. It now follows by Theorem 1.14 with $A=W=H$ and $B=U=K$ that in all these cases $K \subset H$ is $\beta$-Frobenius.

The next result can be considered a "dual version" of Theorem 4.8.

4.10. Theorem. Let $H$ be a Hopf algebra, $K$ a right coideal subalgebra, and $\bar{H}=$ $H / H K^{+}$. Assume $H$ has a right normal basis over $K$ and that $\operatorname{dim} K<\infty$. Then

(a) $\overline{K^{*}}:=K^{*} / K^{*} \cdot K^{+}$is one-dimensional, and

(b) $K$ is a Frobenius algebra.

Proof. First, note that $K \in{ }_{K} \mathcal{M}^{H}$, where the $H$-comodule structure is given by $\triangle: K \rightarrow K \otimes H$. Thus by Lemma $4.7(\mathrm{~b}), K^{*} \in \mathcal{M}_{K}^{H}$, where $K$ acts on $K^{*}$ via $\leftarrow$. Now

$$
\begin{aligned}
K^{*} & \cong \overline{K^{*}} \square \bar{H} H & & \text { by } 4.5(\mathrm{~b}) \text { with } M=K^{*} \\
& \cong \overline{K^{*}} \square \bar{H}(\bar{H} \otimes K) & & \text { by the normal basis isomorphism } \\
& \cong \overline{K^{*}} \otimes K, & &
\end{aligned}
$$

where the isomorphisms are as right $K$-modules. The isomorphism $K^{*} \rightarrow \overline{K^{*}} \otimes K$ is given explicitly by $f \mapsto \sum \overline{f_{0}} \otimes \varphi\left(f_{1}\right)$, where $\varphi: H \rightarrow K$ defines the normal basis isomorphism $H \cong \bar{H} \otimes K$, via $\bar{h} \mapsto \sum \overline{h_{1}} \otimes \varphi\left(h_{2}\right)$.

Since $\operatorname{dim} K=\operatorname{dim} K^{*}<\infty$, clearly $\operatorname{dim} \overline{K^{*}}=1$, proving (a). Since $K^{*} \cong K$ as right $K$-modules, $K$ is Frobenius, proving (b).

All of this section could have been proved on the other side as well. The next corollary will be used for Corollary 5.8, where it is needed on the other side, so we will state and prove it on the other side here.

4.11. Corollary. Let $A$ and $H$ be Hopf algebras and $\pi: A \rightarrow H$ a surjective Hopf algebra map. Consider $A$ as a right $H$-comodule via $\pi$ and define $R:=A^{\text {co } H}$. Assume that $\operatorname{dim} R<\infty$ and that there exists a right $H$-colinear and invertible map $\gamma: H \rightarrow A$. Then 
(a) $R$ is a Frobenius algebra and has non-zero left integrals and right integrals.

(b) Choose $0 \neq t \in \int_{R}^{r}$. Then $\varepsilon(t) \neq 0 \Leftrightarrow R$ is separable.

Proof. (a) More generally, let $A \rightarrow \bar{A}=A / I$ be a quotient right $A$-module and coalgebra and assume there exists $\gamma: \bar{A} \rightarrow A$ which is right $\bar{A}$-colinear and invertible. Let $R:=A^{\text {co } \bar{A}}$, a left coideal subalgebra of $A$, and assume that $\operatorname{dim} R<\infty$. Then by $[\mathrm{MD},(1.4),(1.5)]$,

$$
R \otimes \bar{A} \rightarrow A, \quad \text { given by } r \otimes \bar{a} \mapsto r \gamma(\bar{a}),
$$

is bijective, left $R$-linear, and right $\bar{A}$-colinear. By Lemma 4.3, $I=R^{+} A$, and so Theorem 4.10 (on the other side) applies. Thus $R$ is Frobenius. The algebra $R$ is augmented via the restriction of $\varepsilon_{A}$, so $\int_{R}^{r}$ is one-dimensional since it is the right annihilator of $R^{+}$; similarly for $\int_{R}^{l}$.

(b) If $R$ is separable, then $R \stackrel{\varepsilon}{\rightarrow} k$ splits, as right $R$-modules, and thus $\varepsilon(t) \neq 0$ for $0 \neq t \in \int_{R}^{r}$.

Conversely, we first show that $S_{A}^{2}(R) \subset R$. To see this, first note that $\pi$ is a Hopf algebra map, so in particular $\pi \circ S_{A}=S_{H} \circ \pi$, where $S_{H}$ is the antipode for $H$. Thus, $\forall r \in R$,

$$
(\pi \otimes i d) \triangle S_{A}^{2} r=(\pi \otimes i d) \sum S_{A}^{2} r_{1} \otimes S_{A}^{2} r_{2}=\sum\left(S_{H}\right)^{2} \pi\left(r_{1}\right) \otimes S_{A}^{2} r_{2}=1 \otimes S_{A}^{2} r,
$$

and so $S_{A}^{2}(R) \subset R$

Now part (b) follows from the next lemma, which is $[\mathrm{K}, 5.2]$ on the other side. We give the argument for completeness.

Lemma $[\mathrm{K}]$. Let $R \subset A$ be a finite-dimensional left coideal subalgebra of $A$ such that $S^{2}(R) \subset R$. Let $0 \neq t \in \int_{R}^{r}$ such that $\varepsilon(t)=1$. Then

(a) $\sum r S t_{1} \otimes t_{2}=\sum S t_{1} \otimes t_{2} r$, for all $r \in R$,

(b) $\sum S t_{1} \otimes t_{2} \in R \otimes R$.

In particular, $R$ is separable, since $\sum\left(S t_{1}\right) t_{2}=\varepsilon(t)=1$.

Proof. (a) Indeed,

$$
\begin{aligned}
\sum S t_{1} \otimes t_{2} r & =\sum r_{1} S\left(t_{1} r_{2}\right) \otimes t_{2} r_{3} \\
& =\sum r_{1} S\left(\left(t r_{2}\right)_{1}\right) \otimes\left(t r_{2}\right)_{2} \\
& =\sum r\left(S t_{1}\right) \otimes t_{2},
\end{aligned}
$$

since $t$ is a right integral.

(b) Write $\triangle t=\sum_{i} t_{1, i} \otimes t_{2, i}$, where $\left\{t_{2, i}\right\}$ is a $k$-basis of $R$, and consider $X=$ $\sum k\left(S t_{1, i}\right)$. Then $R X \subset X$ by (a), and also $1 \in X$, since $1=\varepsilon(t)=\sum S\left(t_{1}\left(S t_{2}\right)\right)=$ $\sum^{i} S^{2}\left(t_{2}\right) S t_{1} \in R X$, because $t_{2} \in R$. Hence $R \subset R X \subset X$, and so $R=X$, since $\operatorname{dim} X \leq \operatorname{dim} R$ by construction. Thus $\sum S t_{1} \otimes t_{2} \in R \otimes R$.

\section{Hopf algebras in Yetter-Drinfeld CATEgories}

Let $H$ be a Hopf algebra with a bijective antipode. The Yetter-Drinfeld category ${ }_{H}^{H} \mathcal{Y D}$ is the braided monoidal category whose objects $M$ are both left $H$-modules and left $H$-comodules and satisfy the compatibility condition

$$
\sum h_{1} m_{-1} \otimes h_{2} \cdot m_{0}=\sum\left(h_{1} \cdot m\right)_{-1} h_{2} \otimes\left(h_{1} \cdot m\right)_{0}
$$


for all $h \in H, m \in M$. The braiding $\tau: V \otimes W \mapsto W \otimes V$ in this category is given by

$$
\tau(v \otimes w)=\sum v_{-1} \cdot w \otimes v_{0} .
$$

For details, see $[\mathrm{Y}]$. Now, $R \in{ }_{H}^{H} \mathcal{Y D}$ is a bialgebra in this category if it is both an algebra and a coalgebra, and the bialgebra structure maps are all category morphisms. Moreover, $\triangle_{R}$ must be multiplicative in ${ }_{H}^{H} \mathcal{Y} \mathcal{D}$, using $\tau$ on $R \otimes R$; that is

$$
\triangle_{R}(r s)=\sum r^{1}\left(\left(r^{2}\right)_{-1} \cdot s^{1}\right) \otimes\left(r^{2}\right)_{0} s^{2},
$$

where $\triangle_{R}(r)=\sum r^{1} \otimes r^{2}$ denotes the comultiplication of $R$. For a given $H$, an $R$ which was an algebra and a coalgebra in ${ }_{H} \mathcal{M}$ and in ${ }^{H} \mathcal{M}$, and satisfied the compatibility conditions (5.1) and (5.3), was called admissible in [R 85]. The fact that this is the same as $R$ being a bialgebra in ${ }_{H}^{H} \mathcal{Y D}$ is noted in [Mj]. Given such an $R$, we can form Radford's biproduct $A=R \star H$ [R 85, Theorem 1], which is a usual bialgebra. As an algebra, $A=R \star H$ is the smash product $R \# H$, and as a coalgebra it is the smash coproduct, that is

$$
\triangle_{A}(r \star h)=\sum\left(r^{1} \star\left(r^{2}\right)_{-1} h_{1}\right) \otimes\left(\left(r^{2}\right)_{0} \star h_{2}\right) .
$$

$R$ is a Hopf algebra in ${ }_{H}^{H} \mathcal{Y D}$ if it has an antipode $S_{R}$, that is, a convolution inverse to the identity. $R \star H$ is then a Hopf algebra with antipode given by $S(r \star h)=$ $\sum\left(1 \star S_{H}\left(r_{-1} h\right)\right)\left(S_{R} r_{0} \star 1\right)$ [R 85, Theorem 2]. $S_{R}$ is a map in ${ }_{H}^{H} \mathcal{Y D}$, so it is both $H$ linear and $H$-colinear. For details of these constructions, see also [M, Section 10.6]. Note that under $\triangle_{A}, R$ is a left coideal subalgebra of $A$, but not a subcoalgebra.

Now let $S \subset R$ be an extension of Hopf algebras in the Yetter-Drinfeld category ${ }_{H}^{H} \mathcal{Y D}$. Set $A=W=R \star H$ and $B=U=S \star H, \sigma=\triangle_{A}$ and $\rho=i d \otimes \triangle_{H}$, and assume that $A$ is left or right faithfully flat over $B$. Then $R \cong A^{\text {co } H}$ and $B={ }^{c o} \bar{A} A$, where $\bar{A}=\bar{W}=(R \star H) /(R \star H)(S \star H)^{+}$. In fact $\bar{A} \cong \bar{R}=R / R S^{+}$; see Lemma 5.5 below.

Note that we now have a Hopf algebra surjection $\pi: R \star H \rightarrow H, r \star h \mapsto \varepsilon_{R}(r) h$, and so also have the commutative diagram as in Example 2.7 (b),

$$
\begin{array}{ccccc}
S & \subset & S \star H & \stackrel{\pi}{\rightarrow} & H \\
\cap & & \cap & & \| \\
R & \subset & R \star H & \stackrel{\pi}{\rightarrow} & H
\end{array}
$$

This diagram will be used frequently in the following sections.

Now $S \subset S \star H$ and $R \subset R \star H$ are faithfully flat $H$-Galois extensions, and $k={ }^{c o}{ }^{A} A \subset A$ is $A$-Galois; thus $(S \star H, R \star H, H, R \star H)$ is a faithfully flat bi-Galois extension.

5.5. Lemma. Let $S \subset R$ be an extension of Hopf algebras in the category ${ }_{H}^{H} \mathcal{Y D}$. Then $R S^{+}$is a coideal of $R$. If also $B \subset A$ is left or right faithfully flat, then the inclusion $R \subset A$ induces an isomorphism of coalgebras $\bar{R} \cong \bar{A}$.

Proof. We first check that $R S^{+}$is a coideal of $R$; note that there is something to prove here since $\triangle_{R}$ is not a usual algebra map. Thus, choose $r \in R, s \in S^{+}$. Since $S^{+}$is a coideal of $S$, and $S$ is a subcoalgebra of $R$, we have $\triangle_{R} s \in S \otimes S^{+}+S^{+} \otimes S$. 
Since $S$ is in ${ }_{H}^{H} \mathcal{Y D}$ and $\varepsilon_{S}$ is an $H$-module map, if follows that $H \cdot S^{+} \subseteq S^{+}$. Then

$$
\begin{aligned}
\triangle_{R}(r s) & =\sum r^{1}\left(\left(r^{2}\right)_{-1} \cdot s^{1}\right) \otimes\left(r^{2}\right)_{0} s^{2} \\
& \in \sum r^{1}\left(\left(r^{2}\right)_{-1} \cdot S\right) \otimes\left(r^{2}\right)_{0} S^{+}+\sum r^{1}\left(\left(r^{2}\right)_{-1} \cdot S^{+}\right) \otimes\left(r^{2}\right)_{0} S \\
& \in R \otimes R S^{+}+R S^{+} \otimes R .
\end{aligned}
$$

Thus $R S^{+}$is a coideal and $\bar{R}=R / R S^{+}$is a coalgebra.

Now assume the faithfully flat hypothesis. Then by the above remarks, we are in the situation of 2.7, and thus Proposition 2.8 applies. Consider $R \subset A$ via $r \mapsto r \star 1$. Then

$$
\triangle_{A}(r \star 1)=\sum\left(\left(r^{1} \star 1\right)\left(1 \star\left(r^{2}\right)_{-1}\right)\right) \otimes\left(\left(r^{2}\right)_{0} \star 1\right) .
$$

Under the map $A \rightarrow \bar{A}, r \star h \mapsto(\overline{r \star 1})(\overline{1 \star h)}=\varepsilon(h)(\overline{r \star 1})$. Thus

$$
\begin{aligned}
\triangle_{\bar{A}}(\overline{r \star 1}) & =\sum \varepsilon\left(\left(r^{2}\right)_{-1}\right)\left(\overline{r^{1} \star 1}\right) \otimes\left(\left(\overline{\left.r^{2}\right)_{0} \star 1}\right)\right. \\
& =\sum\left(\overline{r^{1} \star 1}\right) \otimes\left(\overline{r^{2} \star 1}\right) .
\end{aligned}
$$

Thus the inclusion $\bar{R} \subseteq \bar{A}$ is a coalgebra isomorphism.

We write the $H$-comodule structure of $R$ as $\delta_{R}: R \rightarrow H \otimes R, r \mapsto \sum r_{-1} \otimes r_{0}$. We say $S \subset R$ has finite index if $\bar{R}=R / R S^{+}$is finite dimensional.

5.6. Theorem. Let $S \subset R$ be an extension of Hopf algebras of finite index in ${ }_{H}^{H} \mathcal{Y D}$, and let $A=R \star H$ and $B=S \star H$. Assume that $A$ has a right normal basis over $B$ and that $R, S$ and $H$ have bijective antipodes. Then $\int_{\bar{R}^{*}}^{r}=k \lambda$ for some $\lambda \neq 0$, we may choose $\Lambda \in R$ such that $\lambda \leftarrow \Lambda=\varepsilon$, and there exist $\chi_{H} \in \operatorname{Alg}(H, k)$ and $\chi \in \operatorname{Alg}(S, k)$ such that

$$
\lambda(\overline{h \cdot r})=\chi_{H}(h) \lambda(\bar{r}) \quad \text { and } \quad \lambda(\overline{s r})=\chi(s) \lambda(\bar{r}),
$$

for all $r \in R, s \in S$, and $h \in H$.

Consequently $S \subset R$ is $\beta$-Frobenius, where $\beta: S \rightarrow S$ and the Frobenius homomorphism $f: R \rightarrow S$ are given by

$$
\begin{aligned}
& \beta(s):=\sum \chi\left(s^{1}\right) \chi_{H}\left(\left(s^{2}\right)_{-1}\right)\left(s^{2}\right)_{0}, \\
& f(r):=\sum \lambda\left(\overline{r^{1}}\right) r^{2},
\end{aligned}
$$

for all $s \in S, r \in R$. Moreover, dual bases (with respect to $f$ ) are given by

$$
\left\{\bar{S}_{R}\left(\left(\Lambda^{2}\right)_{0}\right), \quad \chi_{H}\left(\left(\Lambda^{2}\right)_{-1}\right) \bar{S}_{H}\left(\left(\Lambda^{2}\right)_{-2}\right) \cdot \Lambda^{1}\right\}
$$

Proof. Since $A$ has a right normal basis over $B, B \subset A$ is of right integral type, by Corollary 4.9. Since $R \subset A=R \star H$ and $S \subset B$ are faithfully flat, Theorem 3.1 and Corollary 3.3 apply to give $\int_{\bar{A}^{*}}^{r}=\int_{\bar{R}^{*}}^{r}=k \lambda$, for some $\lambda \neq 0$, and $\Lambda \in R, \chi \in$ Alg $(B, k)$, such that $\lambda \leftarrow \Lambda=\varepsilon$ and $\lambda \leftarrow b=\chi(b) \lambda$, for all $b \in B$.

Moreover $S \subset R$ is $\beta$-Frobenius, with $\beta(s):=\sum \chi\left(s_{1}\right) s_{2}$, for all $s \in S$, and the Frobenius homomorphism $f(r):=\sum \lambda\left(\overline{r_{1}}\right) r_{2}$, for all $r \in R$. Dual bases of $B \subset A$ are given by $\left\{\bar{S} \Lambda_{2}, \Lambda_{1}\right\}$.

The difficulty is that these descriptions of $\beta, f$, and the dual bases use $\triangle_{A}$ and not $\triangle_{R}$. To express them in terms of $\triangle_{R}$, we use [R 85, Theorem 3]. That is, $A=R \star H$ has a projection $\pi$ onto $H$, so that there exists $\gamma: H \rightarrow A$, a Hopf algebra map, satisfying $\pi \gamma=i d_{H}$. In our case, clearly $\gamma(h)=1 \star h$; in particular, $\gamma(H) \subset B$. Then $A^{c o H}=R$ is a coalgebra, via

$$
\triangle_{R}(r)=\sum r^{1} \otimes r^{2}:=\sum r_{1} \gamma \pi\left(S_{A} r_{2}\right) \otimes r_{3},
$$


an $H$-module algebra via

$$
h \cdot r:=\sum \gamma\left(h_{1}\right) r \gamma\left(S_{H} h_{2}\right)
$$

and an $H$-comodule algebra via

$$
\sum r_{-1} \otimes r_{0}:=\sum \pi\left(r_{1}\right) \otimes r_{2}
$$

The antipode on $R$ is $S_{R} r:=\sum \gamma \pi\left(r_{1}\right) S_{A} r_{2}$, with inverse $\bar{S}_{R} r=\sum\left(\bar{S}_{A} r_{2}\right) \gamma \pi\left(r_{1}\right)$, where the antipode $S_{A}$ of $A$ is bijective. See [R 85, p. 337].

First note that $\chi_{H}=\chi_{A} \circ \gamma$. Using $\bar{R} \cong \bar{A}=A / A B^{+}$, we get

$$
\begin{aligned}
\lambda(\overline{h \cdot r}) & =\lambda\left(\overline{\sum \gamma\left(h_{1}\right) r \gamma\left(S_{H} h_{2}\right)}\right) \\
& =\lambda\left(\overline{\sum \gamma\left(h_{1}\right) r \varepsilon\left(\gamma\left(S_{H} h_{2}\right)\right)}\right) \quad \text { since } \gamma(H) \subseteq B \\
& =\lambda(\overline{\gamma(h) r}) \\
& =\chi(\gamma(h)) \lambda(\bar{r}) .
\end{aligned}
$$

We can now check the formulas for $\beta$ and $f$. Using $\triangle_{R}$ as above, for all $s \in S$ we have

$$
\begin{aligned}
\sum \chi\left(s^{1}\right) \chi_{H}\left(\left(s^{2}\right)_{-1}\right)\left(s^{2}\right)_{0}=\sum \chi\left(s_{1} \gamma \pi\left(S_{A} s_{2}\right)\right) \chi_{H}\left(\pi s_{3}\right) r_{4} \\
=\sum \chi\left(s_{1}\right) \chi\left(\gamma \pi\left(S_{A} s_{2}\right)\right) \chi \gamma\left(\pi s_{3}\right) s_{4} \\
=\sum \chi\left(s_{1}\right) s_{2}=\beta(s),
\end{aligned}
$$

and for all $r \in R$,

$$
\begin{aligned}
\sum \lambda\left(\overline{r^{1}}\right) r^{2} & =\sum \lambda\left(\overline{r_{1} \gamma \pi\left(S_{A} r_{2}\right)}\right) r_{3} \\
& =\sum \lambda\left(\overline{r_{1} \varepsilon\left(\gamma \pi\left(S_{A} r_{2}\right)\right)}\right) r_{3} \quad \text { as } \gamma(H) \subseteq B \\
& =\sum \lambda\left(\overline{\left.r_{1}\right) r_{2}=f(r) .}\right.
\end{aligned}
$$

Finally, the fact that the dual bases $\left\{\bar{S}_{A} \Lambda_{2}, \Lambda_{1}\right\}$ can be rewritten as the desired elements in the statement of the theorem follows from the next lemma. We would like to thank N. Andruskiewitsch for help in the computations in the lemma.

5.7. Lemma. In $A_{\beta} \otimes_{B} A$, we have, for all $r \in R$,

(a) $\sum \bar{S}_{A} r_{2} \otimes r_{1}=\sum\left(\bar{S}_{A} r_{5}\right) \gamma \pi\left(r_{4}\right) \otimes \chi\left(\gamma \pi\left(r_{3}\right)\right)\left(\gamma \pi\left(\bar{S}_{A} r_{2}\right)\right) r_{1}$.

(b) $\sum \bar{S}_{A} r_{2} \otimes r_{1}=\sum \bar{S}_{R}\left(r^{2}\right)_{0} \otimes \chi_{H}\left(\left(r^{2}\right)_{-1}\right) \overline{S_{H}}\left(\left(r^{2}\right)_{-2}\right) \cdot r^{1}$.

Proof. For (a), since $\gamma(H) \subseteq B$,

$$
\begin{aligned}
\sum \bar{S}_{A} r_{2} \otimes r_{1} & =\sum\left(\bar{S}_{A} r_{4}\right) \gamma \pi\left(r_{3}\right) \gamma \pi\left(\bar{S}_{A} r_{2}\right) \otimes r_{1} \\
& =\sum\left(\bar{S}_{A} r_{4}\right) \gamma \pi\left(r_{3}\right) \otimes \beta^{-1}\left(\gamma \pi\left(\bar{S}_{A} r_{2}\right)\right) r_{1} .
\end{aligned}
$$

Since $\beta^{-1}\left(\gamma \pi\left(\bar{S}_{A} r_{2}\right)\right)=\sum \chi^{-1}\left(\gamma \pi\left(\bar{S}_{A} r_{3}\right)\right) \gamma \pi\left(\bar{S}_{A} r_{2}\right)$, it follows that

$$
\sum \bar{S}_{A} r_{2} \otimes r_{1}=\sum\left(\bar{S}_{A} r_{5}\right) \gamma \pi\left(r_{4}\right) \otimes \chi \gamma \pi\left(r_{3}\right) \gamma(\pi)\left(\bar{S}_{A} r_{2}\right) r_{1}
$$

(b) Since $\triangle_{R} r=\sum r^{1} \otimes r^{2}=\sum r_{1} \gamma \pi\left(S_{A} r_{2}\right) \otimes r_{3}$, it follows that

$$
\sum r^{1} \otimes\left(r^{2}\right)_{-2} \otimes\left(r^{2}\right)_{-1} \otimes\left(r^{2}\right)_{0}=\sum r_{1} \gamma \pi\left(S_{A} r_{2}\right) \otimes \pi\left(r_{3}\right) \otimes \pi\left(r_{4}\right) \otimes r_{5} .
$$

Thus

$$
\begin{aligned}
& \left.\sum \bar{S}_{R}\left(r^{2}\right)_{0} \otimes \chi_{H}\left(\left(r^{2}\right)_{-1}\right) S_{H}\left(\left(r^{2}\right)_{-2}\right)\right) \cdot r^{1} \\
& \quad=\sum\left(\bar{S}_{A} r_{6}\right) \gamma \pi\left(r_{5}\right) \otimes \chi \gamma\left(\pi\left(r_{4}\right)\right) \bar{S}_{H}\left(\pi\left(r_{3}\right)\right) \cdot\left(r_{1} \gamma \pi\left(S_{A} r_{2}\right)\right) \\
& \quad=\sum\left(\bar{S}_{A} r_{7}\right) \gamma \pi\left(r_{6}\right) \otimes \chi \gamma\left(\pi r_{5}\right) \gamma\left(\bar{S}_{H}\left(\pi r_{4}\right)\right) r_{1} \gamma \pi\left(S_{A} r_{2}\right) \gamma\left(S_{H} \bar{S}_{H}\left(\pi r_{3}\right)\right) \\
& =\sum\left(\bar{S}_{A} r_{5}\right) \gamma \pi\left(r_{4}\right) \otimes \chi\left(\gamma \pi\left(r_{3}\right)\right) \gamma \pi\left(\bar{S} r_{2}\right) r_{1} .
\end{aligned}
$$

This finishes the proof of the lemma. 
Note that the hypotheses of Theorem 5.6 are always satisfied if either $A=R \star H$ is finite-dimensional or $A$ is pointed. In either case, $A$ is $\bar{A}$-cleft by [Sch 92] and thus has the normal basis property (see also Corollary 4.9).

As a corollary, we obtain analogues of the Larson-Sweedler results [LS] for ordinary finite-dimensional Hopf algebras.

5.8. Corollary. Let $R$ be a finite-dimensional Hopf algebra in ${ }_{H}^{H} \mathcal{Y D}$. Then $R$ is a Frobenius algebra, and $\int_{R}^{r}$ is one-dimensional. If $0 \neq t \in \int_{R}^{r}$, then $\varepsilon(t) \neq 0 \Leftrightarrow R$ is a separable $k$-algebra.

Proof. Let $A=R \star H$, the biproduct as above; $A$ is a Hopf algebra. Then $\pi$ : $A \rightarrow H$, via $r \star h \mapsto \varepsilon(r) h$, is a surjective Hopf algebra map with $R=A^{\text {co } H}$. Thus $\pi$ is split, via $\gamma: H \rightarrow A, h \mapsto 1 \star h$. The result is now a special case of Corollary 4.11.

In fact Theorem 5.6 gives more information in this case: the Frobenius homomorphism $f: R \rightarrow k$ is given by $f(r)=\sum \lambda\left(r^{1}\right) r^{2}=\lambda(r)$ (since $f(r) \in k$ ), and dual bases for $R$ (with respect to $f$ ) are given by

$$
\left\{\bar{S}_{R}\left(\left(t^{2}\right)_{0}\right), \chi_{H}\left(\left(t^{2}\right)_{-1}\right) \bar{S}_{H}\left(\left(t^{2}\right)_{-2}\right) \cdot t^{1}\right\} \text {. }
$$

We remark that it is quite complicated to prove directly the equations showing that the given elements in Theorem 5.6 are dual bases. The direct proof, entirely inside ${ }_{H}^{H} \mathcal{Y} \mathcal{D}$, is difficult even for the special case above when $R$ is finite-dimensional and $S=k$. If also $H$ is finite-dimensional, then the computations simplify slightly and we have an explicit formula for $\chi_{H}$, as we see next.

5.9. Remark. Let $H$ be finite-dimensional; we give an elementary proof of Corollary 5.8 as well as an explicit formula for $\chi_{H}$. For then $A=R \star H$ is a finitedimensional Hopf algebra, so it is a Frobenius algebra. Since $A$ is free over $R$ on both sides, it follows that $R$ is also Frobenius. If $0 \neq t \in \int_{R}^{r}$ and $u \in \int_{H}^{r}$, then one can check directly that $w=t \star u \in \int_{A}^{r}$, using that $\varepsilon_{R}$ is an $H$-module map.

Now for any $h \in H$,

$$
\alpha_{A}(h) w=h w=\sum\left(h_{1} \cdot t\right) \star h_{2} u=\sum\left(h_{1} \cdot t\right) \alpha_{H}\left(h_{2}\right) \star u,
$$

and thus $\alpha_{A}(h) t=\sum\left(h_{1} \cdot t\right) \alpha_{H}\left(h_{2}\right)$, where $\alpha_{A}$ and $\alpha_{H}$ are the right modular functions for $A$ and $H$, respectively. It follows that $h \cdot t=\chi_{H}(h) t$, where

$$
\chi_{H}=\left.\alpha_{A}\right|_{H} * \alpha_{H}^{-1} \in H^{*} \text {. }
$$

Since $\varepsilon_{R}$ is an $H$-module map, it follows that $\varepsilon_{R}(h \cdot t)=\varepsilon_{H}(h) \varepsilon_{R}(t)=\chi(h) \varepsilon_{R}(t)$. Thus if $\varepsilon_{R}(t) \neq 0$, it follows that $\chi_{H}=\varepsilon_{H}$ and so $t \in R^{H}$. Using this fact, a twisted version of the usual Maschke argument shows that $\sum t_{1} \otimes S t_{2}$ centralizes $R$. To see this, first note that since $R$ is Frobenius, $\int_{R}^{r}$ is one-dimensional, and thus (as for usual Hopf algebras) $t$ is also in $\int_{R}^{l}$ since $\varepsilon(t) \neq 0$. Using (5.3), it then follows that for all $r \in R$,

$$
\begin{aligned}
\triangle_{R} t \otimes r & =\sum \triangle_{R}\left(\varepsilon\left(r^{1}\right) t\right) \otimes r^{2}=\sum \triangle_{R}\left(r^{1} t\right) \otimes r^{2} \\
& =\sum r^{1}\left(\left(r^{2}\right)_{-1} \cdot t^{1}\right) \otimes\left(r^{2}\right)_{0} t^{2} \otimes r^{3} .
\end{aligned}
$$

Also

$(* *) \quad \varepsilon(h) \triangle(t)=\triangle(\varepsilon(h) t)=\triangle_{R}(h \cdot t)=h \cdot \triangle(t)=\sum h_{1} \cdot t^{1} \otimes h_{2} \cdot t^{2}$, 
for all $h \in H$, since $t \in R^{H}$. Now for any $r \in R$, we have

$$
\begin{aligned}
\sum t^{1} \otimes\left(S t^{2}\right) r & =\sum r^{1}\left(\left(r^{2}\right)_{-1} \cdot t^{1}\right) \otimes S\left(\left(r^{2}\right)_{0} t^{2}\right) r^{3} \text { using }(*) \\
& =\sum r^{1}\left(\left(r^{2}\right)_{-2} \cdot t^{1}\right) \otimes S\left(\left(r^{2}\right)_{-1} \cdot t^{2}\right) S\left(\left(r^{2}\right)_{0}\right) r^{3} \\
& =\sum r^{1} t^{1} \otimes\left(S t^{2}\right) \varepsilon\left(\left(r^{2}\right)_{-1}\right) S\left(\left(r^{2}\right)_{0}\right) r^{3} \text { using }(* *) \\
& =\sum r^{1} t^{1} \otimes\left(S t^{2}\right)\left(S r^{2}\right) r^{3} \\
& =\sum r t^{1} \otimes S t^{2} .
\end{aligned}
$$

Since $\sum t_{1} S t_{2}=\varepsilon(t) \neq 0$, it follows that $R$ is separable.

We will see in Example 5.12 that if $R$ is not separable, then $\chi_{H}$ can be non-trivial and $t \notin R^{H}$.

5.10. Example: Graded Hopf algebras. Let $H=k G$, where $G$ is an abelian group with a bicharacter $\langle\mid\rangle: G \times G \rightarrow k^{*}$, and consider the category of $k G$ comodules ${ }^{k G} \mathcal{M}$. Recall that ${ }^{k G} \mathcal{M}$ is just the category of $G$-graded $k$-modules; moreover it is contained in the Yetter-Drinfeld category ${ }_{H}^{H} \mathcal{Y D}$, for $H=k G$, since any $M \in{ }^{k G} \mathcal{M}$ is also a left $k G$-module via $g \cdot m_{h}=\langle g \mid h\rangle m_{h}$, for all $g, h \in G$ and $m_{h} \in M_{h}$, the $h$-component of $M$. Then for $G$-graded modules $V$ and $W$, and homogeneous elements $v \in V_{g}$ and $w \in W_{h}$, the twist is given by

$$
\tau: V \otimes W \rightarrow W \otimes V, \text { via } v \otimes w \mapsto\langle g \mid h\rangle w \otimes v .
$$

For $A$ and $B G$-graded algebras, $A \otimes B$ becomes an associative algebra via

$$
\left(m_{A} \otimes m_{B}\right) \circ(i d \otimes \tau \otimes i d): A \otimes B \otimes A \otimes B \rightarrow A \otimes B .
$$

$R$ is a $G$-graded Hopf algebra if it is both a $G$-graded algebra and a $G$-graded coalgebra, if $\triangle_{R}$ is multiplicative in ${ }^{k G} \mathcal{M}$ using the above multiplication in $A \otimes A$, and if the antipode $S$ is a map in ${ }^{k G} \mathcal{M}$. It will then follow that the biproduct $A=R \star k G$ is an ordinary Hopf algebra. If $S \subset R$ are $G$-graded Hopf algebras, diagram (5.4) becomes

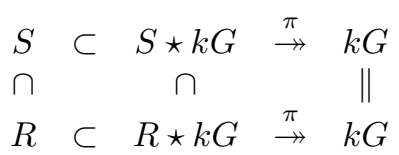

As a special case, we will consider extensions $U(K) \subset U(L)$ of $G$-Lie coloralgebras in Section 6. We also consider an old example of Radford:

5.11. Radford's example revisited. We reconsider the example of Radford [R 85, Section 4]. We first rewrite it in the language of graded Hopf algebras, which we believe simplifies the exposition, and then compute for it the data in Theorem 5.6. Let $G=\mathbb{Z}_{n}$, and assume that the base field $k$ contains a primitive $n^{\text {th }}$ root of 1 , say $\omega$. Define

$$
\langle\mid\rangle: \mathbb{Z}_{n} \times \mathbb{Z}_{n} \rightarrow k^{*} \quad \text { by }\langle i \mid j\rangle=\omega^{i j},
$$

for all $i, j \in \mathbb{Z}_{n}$. Then $\langle\mid\rangle$ is a bicharacter on $\mathbb{Z}_{n}$, so as above it determines a braiding on the category of $\mathbb{Z}_{n}$-graded modules: if $M=\bigoplus_{i \in \mathbb{Z}_{n}} M_{i}$ and $N=$ $\bigoplus_{j \in \mathbb{Z}_{n}} N_{j}$, and $m_{i} \in M_{i}, n_{j} \in N_{j}$, then

$$
\tau: M \otimes N \rightarrow N \otimes M \quad \text { is given by } \quad m_{i} \otimes n_{j} \mapsto \omega^{i j}\left(n_{j} \otimes m_{i}\right) .
$$


Radford's example (in the case $m=1$ ) is defined to be the $k$-algebra

$$
\left.R=k\left\langle u_{0}, \cdots, u_{n-1}\right| u_{i} u_{j}=0, i \neq j, \quad \text { and } \quad u_{i}^{n+1}=\alpha_{i} u_{i}, i=0, \cdots, n-1\right\rangle
$$

where the scalars $\alpha_{i} \in k^{*}$ will be determined below. Note that for all $i, e_{i}=\alpha_{i}^{-1} u_{i}^{n}$ is an idempotent and $1_{R}=\sum_{i=0}^{n-1} e_{i}$. Thus as an algebra, $R=\bigoplus_{i=0}^{n-1} S_{i}$, where $S_{i}=$ $k$-span $\left\{u_{i}^{j} \mid j=1, \cdots, n\right\}$, and so $\operatorname{dim} R=n^{2}$. If also $\alpha_{i}^{-1 / n}=\beta_{i} \in k$, then $\left(\beta_{i} u_{i}\right)^{n}=e_{i}$ and so $S_{i} \cong k \mathbb{Z}_{n}$, for all $i$. $R$ becomes a $\mathbb{Z}_{n}$-graded algebra by defining degree $\left(u_{i}\right):=i$. That is, $R=\bigoplus_{i \in \mathbb{Z}_{n}} R_{i}$, with $R_{i} R_{j} \subseteq R_{i+j(\bmod n)}$, where $R_{i}=$ $k$-span $\left\{u_{j}^{k} \mid k j \equiv i(\bmod n)\right\}$.

We next consider $R$ as a graded coalgebra. Define

$$
\triangle u_{i}=\sum_{l=0}^{n-1} u_{l} \otimes u_{i-l} \quad \text { and } \quad \varepsilon\left(u_{i}\right)=\delta_{0, i},
$$

for all $i=0, \cdots, n-1$. Thus $\triangle u_{i} \in \sum_{l} R_{l} \otimes R_{i-l}$. We define $\triangle\left(u_{i}^{k}\right)$ so that $\triangle$ becomes a graded-multiplicative map on $R$. Using induction, this means that

$$
\begin{aligned}
\triangle\left(u_{i}^{k}\right) & :=\left(\triangle u_{i}\right)^{k} \quad(\text { where we use the graded product on } R \otimes R) \\
& =\sum_{l=0}^{n-1} \omega^{l(i-l)(1+2+\cdots+(k-1))} u_{l}^{k} \otimes u_{i-l}^{k} \\
& =\sum_{l=0}^{n-1} \omega^{l(i-l) \frac{k(k-1)}{2}} u_{l}^{k} \otimes u_{i-l}^{k} .
\end{aligned}
$$

For $k=n+1,(*)$ must be compatible with the relations $u_{i}^{n+1}=\alpha_{i} u_{i}$ in $R$. Solving, we see that a necessary and sufficient condition on the $\alpha_{i}$ for $\triangle$ to be graded-multiplicative is that

$$
\alpha_{i}=\left(\omega^{\frac{n(n+1)}{2}}\right)^{l(i-l)} \alpha_{l} \alpha_{i-l},
$$

for all $i, l=0, \cdots, n-1$. Now if $n$ is odd, $\omega^{\frac{n(n+1)}{2}}=1$ and if $n$ is even, $\omega^{\frac{n(n+1)}{2}}=-1$. Summarizing, we have:

Proposition. With generators and relations as above, $R$ is a $\mathbb{Z}_{n}$-graded bialgebra provided we can find $\alpha_{i} \in k^{*}$ such that

(1) $\alpha_{i}=\alpha_{l} \alpha_{i-l}$, all $i, l$, if $n$ is odd, or

(2) $\alpha_{i}=(-1)^{l(i-l)} \alpha_{l} \alpha_{i-l}$, all $i, l$ if $n$ is even.

Moreover $R \cong\left(k \mathbb{Z}_{n}\right)^{(n)}$ as an algebra if $\alpha_{i}^{1 / n} \in k$, for all $i$.

Example. If $n$ is odd, condition (1) is satisfied by setting $\alpha_{i}=\omega^{i}$. If $n$ is even and $k$ contains a primitive $4^{t h}$ root of 1 , condition (2) is satisfied by setting $\alpha:=$ $(-1)^{\frac{i(n-i)}{2}} \omega^{i}$. We continue with these assumptions on $k$ and the $\alpha_{i}$.

Now $R$ becomes a $\mathbb{Z}_{n}$-graded Hopf algebra by defining $S_{R} u_{i}=\alpha_{n-i}^{-1} u_{n-i}^{n-1}$; note that $S_{R} u_{i} \subseteq R_{i}$ since $(n-1)(n-i) \equiv i(\bmod n) . S_{R}$ is extended to $R$ as a graded (anti-) homomorphism; then for $1 \leq k \leq n$

$$
S_{R}\left(u_{i}^{k}\right)=\left(\alpha_{n-1}\right)^{-1} \omega^{i^{2}\left(\begin{array}{c}
k \\
2
\end{array}\right)} u_{n-i}^{n-k} .
$$


We check that $S_{R} * i d=\varepsilon$ on $u_{i}$ :

$$
\begin{aligned}
\left(S_{R} * i d\right)\left(u_{i}\right) & =\sum_{l=0}^{n-1}\left(S u_{l}\right) u_{i-l}=\sum_{l=0}^{n-1} \alpha_{n-l}^{-1} u_{n-l}^{n-1} u_{i-l}=\left\{\begin{array}{lll}
0 & \text { if } i \neq 0 \\
1 & \text { if } i=0
\end{array}\right. \\
& =\delta_{i, 0}=\varepsilon\left(u_{i}\right) .
\end{aligned}
$$

Radford shows that $S_{R}$ has order $2 n$. However in the biproduct $A=R \star H=$ $R \# k \mathbb{Z}_{n}$ we have $S_{A}^{2}=i d$, and thus $A$ is involutory.

Thus we have constructed a semisimple graded Hopf algebra which is not a usual Hopf algebra, since $\triangle_{R}$ is not an algebra map in the usual sense. Such an example is not possible if we use $R=u(L)$, the restricted enveloping algebra of a restricted Lie superalgebra $L=L_{0} \oplus L_{1}$, which is a $\mathbb{Z}_{2}$-graded Hopf algebra. For in that case, it is shown in [Be] that $u(L)$ is semisimple only if $L_{1}=0$; that is, $u(L)$ is an ordinary Hopf algebra.

We now turn to the data in Corollary 5.4 and Theorem 5.6. First, it is easy to see that $t=\sum_{k=1}^{n} u_{0}^{k}$ is a two-sided integral for $R$, since $t u_{j}=0=\varepsilon\left(u_{j}\right)$ if $j \neq 0$, and $t u_{0}=t=\varepsilon\left(u_{0}\right) t$ (since $\alpha_{0}=1$ implies $\left.u_{0}^{n+1}=u_{0}\right)$. Now $\varepsilon(t)=\sum_{k=1}^{n} \varepsilon\left(u_{0}\right)^{k}=n \neq 0$ in $k$, as predicted since $R$ is semisimple.

Next we compute the dual bases for $R$. In this case, the formula in Theorem 5.6 simplifies considerably. For, we may choose $\Lambda$ to be any (right) integral in $R$; thus let $\Lambda=t$ as above. Since $R$ is semisimple, $\chi_{H}=\varepsilon$ from Remark 5.9; thus the dual bases after Corollary 5.8 become

$$
\left\{\overline{S_{R}}\left(\left(t^{2}\right)_{0}\right), \overline{S_{H}}\left(\left(t^{2}\right)_{-1}\right) \cdot t^{\prime}\right\}
$$

More specifically,

$$
\triangle_{R} t=\sum_{k=1}^{n} \triangle_{R}\left(u_{0}^{k}\right)=\sum_{k} \sum_{l=0}^{n-1} \omega^{-l^{2}\left(\begin{array}{c}
k \\
2
\end{array}\right)} u_{l}^{k} \otimes u_{n-l}^{k} .
$$

Since $\bar{S}$ is the composition inverse of $S$, it is easy to see that

$$
\bar{S}\left(u_{n-i}^{k}\right)=\alpha_{n-i} \omega^{-i^{2}\left(\begin{array}{c}
k \\
2
\end{array}\right)} u_{i}^{n-k} .
$$

For $t^{2}=u_{n-l}^{k} \in R_{-l k}$, we have $\left(t^{2}\right)_{0}=t^{2}$ and $\left(t^{2}\right)_{-1}=-l k$. Then the dual bases for $R$ are

$$
\left\{\alpha_{n-i} \omega^{-i^{2}\left(\begin{array}{c}
n \\
2
\end{array}\right)}+u_{i}^{n-k}, u_{i}^{k}\right\} \text {. }
$$

5.12. Example. Again we consider a $\mathbb{Z}_{n}$-graded Hopf algebra, with the same bicharacter on $\mathbb{Z}_{n}$ as in Example 5.11, and thus the same braiding. Let

$$
R=k\left[x \mid x^{n}=0\right] .
$$

$R$ is a $\mathbb{Z}_{n}$-graded algebra by setting $R_{i}=k x^{i}$, and it is also a $\mathbb{Z}_{n}$-graded coalgebra by setting $\triangle_{R} x=x \otimes 1+1 \otimes x$ and $\varepsilon(x)=0$ and extending multiplicatively using the twist map. Thus

$$
\triangle_{R} x^{k}=\sum_{i=0}^{k}\left[\begin{array}{l}
k \\
i
\end{array}\right]_{q} x^{i} \otimes x^{k-i},
$$

where $\left[\begin{array}{l}k \\ i\end{array}\right]_{q}$ is the $q$-binomial coefficient using $q=\omega . R$ is a $\mathbb{Z}_{n}$-graded Hopf algebra, with

$$
S_{R}\left(x^{k}\right)=(-1)^{k} \omega^{\left(\begin{array}{c}
k \\
2
\end{array}\right)} x^{k} .
$$


It is easy to see that $t=x^{n-1}$ is a left and right integral for $R$. As in Example $5.10, \mathbb{Z}_{n}$ acts on $r_{\bar{j}} \in R_{\bar{j}}$ via $\bar{i} \cdot r_{\bar{j}}=\langle i \mid j\rangle r_{\bar{j}}=\omega^{i j} r_{\bar{j}}$. Then $\bar{i} \cdot t=\omega^{i(n-1)} t=\omega^{-i} t$. Thus using Remark 5.9, we see that $\chi_{H}(\bar{i})=\omega^{-i}$. In particular, $\chi_{H} \neq \varepsilon_{H}$ and $t \notin R^{G}$.

Using the comment after Corollary 5.8, we can find dual bases for $R$. Since

$$
\triangle_{R} t=\triangle_{R} x^{n-1}=\sum_{i=0}^{n-1}\left[\begin{array}{c}
n-1 \\
i
\end{array}\right]_{q} x^{i} \otimes x^{n-1-i}
$$

and since $x^{i} \in R_{\bar{i}}$, the dual bases are

$$
\left\{\bar{S}_{R}\left(x^{n-1-i}\right), \chi_{H}(\overline{n-1-i}) \bar{S}_{H}(\overline{n-1-i}) \cdot\left[\begin{array}{c}
n-1 \\
i
\end{array}\right]_{\omega} x^{i}\right\}
$$

for all $i$. Simplifying, the right-hand terms become

$$
\left[\begin{array}{c}
n-1 \\
i
\end{array}\right]_{\omega} \omega^{i+1} \overline{i+1} \cdot x^{i}=\left[\begin{array}{c}
n-1 \\
i
\end{array}\right]_{\omega} \omega^{i+1} \omega^{-(i+1) i} x^{i}=\left[\begin{array}{c}
n-1 \\
i
\end{array}\right]_{\omega} \omega^{-i^{2}+1} x^{i} .
$$

Together with the above formulas for $\bar{S}$ and the action, this gives the dual bases:

$$
\left\{(-1)^{n-i-1} \omega^{-\left(\begin{array}{c}
n-i-1 \\
2
\end{array}\right)} x^{n-i-1},\left[\begin{array}{c}
n-1 \\
i
\end{array}\right]_{\omega} \omega^{-i^{2}+1} x^{i}\right\} .
$$

We remark that in this case, $A=R \star H$ is just the Taft algebra of dimension $n^{2}$ [Tf]. For, writing $\mathbb{Z}_{n}=\langle g\rangle$ multiplicatively, we have $g \cdot x=g x g^{-1}=\omega^{-1} x$, or $x g=\omega g x$. In the Hopf algebra $A, g \in G(A)$ and $\triangle_{A} x=x \otimes g+1 \otimes x$.

\section{Lie SUPERALGEBRAS AND LIE COLORALGEBRAS}

We now apply our main theorem to recover the result of Bell and Farnsteiner $[\mathrm{BF}]$ concerning when an extension $U(K) \subset U(L)$ is $\beta$-Frobenius, where $K \subset L$ are Lie superalgebras. Moreover, with very little extra work, we generalize their result to enveloping algebras of Lie coloralgebras. For basic facts on Lie coloralgebras, see [Sche] or [BMPZ].

Thus as in Example 5.10, let $H=k G$, where $G$ is an abelian group (written multiplicatively) and $\langle\mid\rangle: G \times G \rightarrow k^{*}$ is a symmetric bicharacter; that is, $\langle\mid\rangle$ is a bi-homomorphism and satisfies $\langle g \mid h\rangle^{-1}=\langle h \mid g\rangle$, for all $g, h \in G$. Then a $G$-Lie coloralgebra with respect to $\langle\mid\rangle$ is a $G$-graded vector space $L=\bigoplus_{g \in G} L_{g}$ with a $G$-graded $k$-linear binary operation [ , ] $L \otimes L \rightarrow L$ such that for any $x \in L_{g}, y \in L_{h}, z \in L_{l}$,

(1) $[x, y]=-\langle g \mid h\rangle[y, x]$, and

(2) $\langle l \mid g\rangle[x,[y, z]]+\langle h \mid l\rangle[z,[x, y]]+\langle g \mid h\rangle[y,[z, x]]=0$. These are the graded ver-

sions of anti-symmetry and the Jacobi identity, respectively. A universal enveloping algebra $U(L)$ of $L$ exists [Sche], and a PBW theorem holds for $U(L)$. However, to describe it, we need more notation.

First, by the symmetry of $\langle\mid\rangle$, we have $\langle g \mid g\rangle= \pm 1$ for each $g \in G$, and thus $G=G_{+} \cup G_{-}$, where

$$
G_{+}=\{g \in G \mid\langle g \mid g\rangle=1\} \text { and } G_{-}=\{g \in G \mid\langle g \mid g\rangle=-1\} .
$$

Then $L=L_{+} \oplus L_{-}$, where $L_{+}=\bigoplus_{g \in G_{+}} L_{g}$ and $L_{-}=\bigoplus_{g \in G_{-}} L_{-}$; note that $\left[L_{+}, L_{+}\right] \subseteq$ $L_{+}$and that $\left[L_{-}, L_{-}\right] \subseteq L_{+}$. 
For the special case of Lie superalgebras, in which $G=\mathbb{Z}_{2}=\{\overline{0}, \overline{1}\}$, the bicharacter is given by $\langle g \mid h\rangle=(-1)^{g h}$; note that here we write $G$ under addition. Thus $G_{+}=\{\overline{0}\}$ and $G_{-}=\{\overline{1}\}$, and so $L_{+}=L_{0}$, which is an ordinary Lie algebra, and $L_{-}=L_{1}$.

In general a $G$-homogeneous basis of $L$ can be written as $X=X_{+} \cup X_{-}$, where $X_{+}$is a basis of $L_{+}$and $X_{-}$is a basis of $L_{-}$; in a total ordering of $X$, we assume that $y<x$ for all $y \in X_{+}$and $x \in X_{-}$.

6.1. PBW Theorem ([BMPZ, p.85]). Assume that char $k \neq 2,3$ and let $X=$ $X_{+} \cup X_{-}$be a $G$-homogeneous basis of the $G$-Lie coloralgebra $L$. Then the universal enveloping algebra $U(L)$ has a $G$-homogeneous basis over $k$ consisting of 1 and all monomials of the form

$$
y_{i_{1}}^{n_{1}} y_{i_{2}}^{n_{2}} \cdots y_{i_{k}}^{n_{k}} x_{j_{1}} x_{j_{2}} \cdots x_{j_{l}}
$$

where $y_{i_{1}}<y_{i_{2}}<\cdots<y_{i_{k}}$ in $X_{+}$and $x_{j_{1}}<x_{j_{2}}<\cdots<x_{j_{l}}$ in $X_{-}$, and $n_{i} \geq 0$.

For the rest of this section, assume that char $k \neq 2,3$ and that $K \subset L$ are $G$-Lie coloralgebras. Since we wish to consider the situation when $[U(L): U(K)]<\infty$, it follows from the PBW theorem that we must assume that $L_{+} \subset K \subset L$, or equivalently that $K_{+}=L_{+}$, and that $[L: K]<\infty$. We now follow the notation in $[\mathrm{BF}]$, used there for Lie superalgebras.

Fix homogeneous elements $x_{1}, \cdots, x_{n} \in L_{-}$whose cosets $\left\{x_{i}+K\right\}$ form a basis of $L / K$; note that $[L: K]=n$. Let $\mathcal{F}=\{0,1\}^{n}$ be the set of multi-indices of length $n$; in particular let $\mathbf{1}=(1,1, \cdots, 1)$ and $\mathbf{0}=(0, \cdots, 0)$. For any $I=(i(1), \cdots, i(n)) \in$ $\mathcal{F}$, we define

$$
\mathbf{x}^{I}=x_{1}^{i(1)} \cdots x_{n}^{i(n)}
$$

thus $\mathbf{x}^{0}=1$ and $\mathbf{x}^{1}=x_{1} x_{2} \cdots x_{n}$. Also define $|I|:=\sum_{l=1}^{n} i(l)$, the weight of $I ; J \leq I$ if $j(l) \leq i(l)$ for all $l=1, \cdots, n$. By the PBW theorem, every element $u$ of $U(L)$ can be written uniquely as

$$
u=\sum_{I \in \mathcal{F}} u_{I} \mathbf{x}^{I}
$$

where $u_{I} \in U(K)$. $U(L)$ has a filtration via $V(m):=\sum_{i=0}^{m} U(K)\left(L_{-}\right)^{i}$; note that $V(n)=U(L) . V(m)$ is also a right $U(K)$-module, and again by PBW,

$$
V(m)=\bigoplus_{|I| \leq m} U(K) \mathbf{x}^{I}=\bigoplus_{|I| \leq m} \mathbf{x}^{I} U(K) .
$$

We now put this setup into the format of Theorem 5.6. The Hopf algebra in question is $H=k G$. $H$ acts on $U(L)$ as in Example 5.10: for any $g \in G$ and homogeneous element $z \in U(L)_{h}, g \cdot z=\langle g \mid h\rangle z$. Thus in the smash product $U(L) \star H,(1 \star g)(z \star 1)=(g \cdot z) \star g=\langle g \mid h\rangle z \star g$. We abbreviate this by $g z=\langle g \mid h\rangle z g$. The diagram of 5.10 becomes

$$
\begin{array}{ccccc}
S=U(K) & \hookrightarrow & B=U(K) \star k G & \stackrel{\pi}{\rightarrow} & k G \\
\cap & & & \cap & \| \\
R=U(L) & \hookrightarrow & A=U(L) \star k G & \stackrel{\pi}{\rightarrow} & k G .
\end{array}
$$

By Lemma 5.5, we know that $\bar{A}=A / A B^{+} \cong \bar{R}=R / R S^{+}$as coalgebras. Moreover $\operatorname{dim}_{k} \bar{A}=\operatorname{dim}_{k} \bar{R}=2^{n}$, and $\bar{A} \cong \bar{R}$ has $k$-basis $\left\{\overline{\mathbf{x}^{I}} \mid I \in \mathcal{F}\right\}$. Since the coalgebra 
structure of $\bar{R}$ is induced from that of $R, \triangle_{R} z=z \otimes 1+1 \otimes z$ for each $z \in L$, and $\triangle_{R}$ is graded-multiplicative, it follows that

$$
\triangle \overline{\mathbf{x}^{I}}=\sum_{J \leq I} \alpha_{I, J} \overline{\mathbf{x}^{J}} \otimes \overline{\mathbf{x}^{I-J}}
$$

for some scalars $\alpha_{I, J} \in k$. Now $\bar{A}^{*}$ has a basis $\left\{f_{I} \mid I \in \mathcal{F}\right\}$ dual to the $\left\{\overline{\mathbf{x}^{I}}\right\}$; that is, $f_{I}\left(\overline{\mathbf{x}^{J}}\right)=\delta_{I, J}$.

6.2. Proposition. The extension $U(K) \star k G \subset U(L) \star k G$ is of (right) integral type. The data $\lambda$ and $\chi$ of Definition 1.10 may be constructed explicitly as follows:

(a) $\lambda \in I_{r}\left(\bar{A}^{\star}\right)$ is given by $\lambda=f_{\mathbf{1}}$.

(b) To define $\chi \in B^{\star}$, we proceed as follows:

(i) Define $\overline{a d}: K \rightarrow g l(L / K) \cong g l_{n}(k)$ by $\overline{a d}(b)(y+K):=a d(b)(y)+K=$ $[b, y]+K$, for $b \in K, y \in L$. Now let

$$
\chi(b):= \begin{cases}\operatorname{tr}(\overline{a d}(b)) & \text { for } b \in K_{1}, \\ 0 & \text { for } b \in K_{g}, g \neq 1 .\end{cases}
$$

$\chi$ is a morphism of Lie coloralgebras, and so extends to a homomorphism $U(K) \rightarrow k$.

(ii) For $g \in G$, define

$$
\chi(g):=\left\langle g \mid g_{1} g_{2} \cdots g_{n}\right\rangle,
$$

where the $x_{i} \in L_{g_{i}}$, for $g_{i} \in L_{-}$, are the homogeneous basis of $L / K$ fixed above.

Proof. (a) First, since $U(L) \star k G$ is pointed, the extension is of right integral type by Corollary 4.9. The algebra structure of $\bar{A}^{*}$ is given by convolution, determined by $\triangle$ (given above) on $\bar{A}=\bar{R}$. Define $\tilde{f}_{i} \in \bar{R}^{*}$ by $\tilde{f}_{i}=f_{I}$, for $I=(0, \cdots, 1,0, \cdots, 0)$; that is, with a 1 only in the $i^{\text {th }}$ position. One may check that $\tilde{f}_{i}^{2}=0$, for all $i$, and that $\tilde{f}_{i} \tilde{f}_{j}=\left\langle g_{j} \mid g_{i}\right\rangle \tilde{f}_{j} \tilde{f}_{i}=f_{(i+j)}$, for $i<j$, where $I=(i+j)$ has a 1 in the $i^{\text {th }}$ and $j^{\text {th }}$ position. It follows that

$$
\left.\bar{R}^{*}=\bar{A}^{*}=k\left\langle\tilde{f}_{1}, \cdots, \tilde{f}_{n}\right| \tilde{f}_{i}^{2}=0 \text { for all } i, \tilde{f}_{i} \tilde{f}_{j}=\left\langle g_{j} \mid g_{i}\right\rangle \tilde{f}_{j} \tilde{f}_{i} \text { for all } i \neq j\right\rangle
$$

with identity element $\bar{\varepsilon}$. $\bar{A}^{*}$ is augmented via evaluation at $\overline{1}$; thus $\left\langle\tilde{f}_{i}, \overline{1}\right\rangle=0$, for all $i$. It is now straightforward to see that $f_{1}$ is a right integral in $\bar{A}^{*}$.

(b) We must check that for all $b \in B$ and $w \in W=A, \lambda(\overline{b w})=\chi(b) \lambda(\bar{w})$. First, since $\bar{A}$ is a left $A$-module, it suffices to check this for $w$ such that $\bar{w}$ is a basis element of $\bar{A}$, that is, $w=\mathbf{x}^{I}$, for some $I$. Moreover, we may assume that $b \in U(K)$, for if $b=z \star g$, where $z \in U(K)$ and $g \in G$, then $g w \in k w g$ and thus $\overline{b w}=\overline{(z \star g) w} \in k \overline{z w}$.

If $|I|<n$, then $b w \in V(m)=\bigoplus_{|J|<m} \mathbf{x}^{J} U(K)$ for $m<n$, and so $\lambda(\overline{b w}) \in$ $\sum_{|J| \leq m} \lambda\left(\mathbf{x}^{J} U(K)\right)$. However if $c \in U(K)$, then $\overline{\mathbf{x}^{J} c}=\varepsilon(c) \overline{\mathbf{x}^{J}}$ and thus $\lambda\left(\overline{\mathbf{x}^{J}}\right)=0$ by (1), since $\lambda=f_{\mathbf{1}}$ and $|J|<n$. Thus $\lambda(\overline{b w})=0=\chi(b) \lambda(\bar{w})$ and there is nothing to prove.

We may therefore assume that $|I|=n$ and that $w=x_{1} x_{2} \cdots x_{n}=\mathbf{x}^{1}$. First consider the case that $a=g \in G$. Then, assuming $x_{i} \in L_{g_{i}}$,

$$
\begin{aligned}
g x_{1} \cdots x_{n} & =\left\langle g \mid g_{1}\right\rangle x_{1} g x_{2} \cdots x_{n}=\cdots \\
& =\left\langle g \mid g_{1}\right\rangle\left\langle g \mid g_{2}\right\rangle \cdots\left\langle g \mid g_{n}\right\rangle x_{1} x_{2} \cdots x_{n} g \\
& =\left\langle g \mid g_{1} g_{2} \cdots g_{n}\right\rangle x_{1} \cdots x_{n} g .
\end{aligned}
$$


Thus

$$
\begin{aligned}
\lambda(\overline{g w}) & =\left\langle g \mid g_{1} \cdots g_{n}\right\rangle \lambda\left(\overline{x_{1} \cdots x_{n} g}\right) \\
& =\left\langle g \mid g_{1} \cdots g_{n}\right\rangle \lambda(\bar{w})=\chi(g) 1,
\end{aligned}
$$

since $g \in k G \subset U(K) \star k G$ and $\varepsilon(g)=1$. This shows (ii).

Now assume that $b \in K$; in fact we may assume that $b$ is homogeneous, say $b \in K_{g}$. It follows that

$(*)$

$$
\begin{aligned}
b x_{1} x_{2} \cdots x_{n}= & {\left[b, x_{1}\right] x_{2} \cdots x_{n}+\left\langle g \mid g_{1}\right\rangle x_{1}\left[b, x_{2}\right] \cdots x_{n} } \\
& +\cdots+\left\langle g \mid g_{1} \cdots g_{n-1}\right\rangle x_{1} \cdots x_{n-1}\left[b, x_{n}\right]+\left\langle g \mid g_{1} \cdots g_{n}\right\rangle x_{1} \cdots x_{n} b .
\end{aligned}
$$

This relation is easy to check using $\left[b, x_{i}\right]=b x_{i}-\left\langle g \mid g_{i}\right\rangle x_{i} b$. Note that $\overline{x_{1} \cdots x_{n} b}=$ $\varepsilon(b) \overline{x_{1} \cdots x_{n}}=\overline{0}$ and thus we may ignore the last term. If $b \in K_{-}$, then $\left[b, x_{i}\right] \in$ $\left[L_{-}, L_{-}\right] \subseteq L_{+}=K_{+}$. Thus each term

$$
x_{1} \cdots x_{i}\left[b, x_{i+1}\right] \cdots x_{n} \in V(n-1)
$$

and so we see immediately that $\lambda\left(\overline{b x_{1} \cdots x_{n}}\right)=0$. This agrees with our definition of $\chi(b)=0$ for $b \notin K_{1}$.

We may therefore assume that $b \in K_{+}$. Now consider the map $\phi: K \rightarrow g l(L / K)$ given by $\phi(b)\left(x_{i}+K\right)=\left[b, x_{i}\right]+K$, and write $\left[b, x_{i}\right]=\sum_{j} \alpha_{i j} x_{j}+K$; that is, $\phi(b)$ has matrix $\left[\alpha_{i j}\right]$. Now for any $c \in K, \overline{x_{1} \cdots x_{i} c x_{i+2} \cdots x_{n}} \in \overline{V(n-1)}$, and we know $\lambda(\overline{V(n-1)})=0$ by the above. Similarly, since $x_{i}^{2} \in K$ for all $i$, and $\left[x_{i}, x_{j}\right] \in\left[L_{-}, L_{-}\right] \subseteq L_{+}=K_{+}$, any monomial $m$ obtained from $w$ by replacing some $x_{k}$ by $x_{i}, k \neq i$, satisfies $m \equiv 0 \bmod V(n-2) \subset V(n-1)$, and thus $\lambda(\bar{m})=0$. Using these observations in $(*)$, we see that

$$
\lambda\left(\overline{b x_{1} \cdots x_{n}}\right)=\left(\alpha_{11}+\left\langle g \mid g_{1}\right\rangle \alpha_{22}+\cdots+\left\langle g \mid g_{1} \cdots g_{n-1}\right\rangle \alpha_{n n}\right) \lambda\left(\overline{x_{1} \cdots x_{n}}\right) .
$$

Now if $g \neq 1$, then $\left[b, x_{i}\right] \in L_{g_{i}} \neq L_{g_{i}}$, and so $\alpha_{i i}=0$, for all $i=1, \cdots, n$. It follows that $\lambda\left(\overline{b x_{1} \cdots x_{n}}\right)=0=\chi(b) 1$. Thus we may assume $g=1$. But now $\langle g \mid h\rangle=1$ for all $h \in G$, by properties of the bicharacter, and so

$$
\lambda\left(\overline{b x_{1} \cdots x_{n}}\right)=\left(\alpha_{11}+\alpha_{22}+\cdots+\alpha_{n n}\right) \lambda(\bar{w})=\chi(b) 1 .
$$

Thus $\chi(b)=\operatorname{trace}(\overline{a d}(b))$, proving (i).

We note that the remaining piece of data in Definition 1.10, namely $\Lambda$, does not seem to be easy to compute explicitly. This is analogous to the difficulty of computing the integral in a restricted enveloping algebra of a (usual) restricted Lie algebra.

6.3. Corollary. Assume $K \subset L$ are Lie coloralgebras such that $K_{+}=L_{+}$and $[L: K]<\infty$. Then $U(K) \subset U(L)$ is a $\beta$-Frobenius extension. The Frobenius homomorphism $f: U(L) \rightarrow U(K)$ is given by $f(r)=\sum f_{\mathbf{1}}\left(\overline{r^{1}}\right) r^{2}$, for all $r \in U(L)$, where $\triangle_{U(L)}(r)=\sum r^{1} \otimes r^{2}$ and $f_{\mathbf{1}}$ is the integral in $\overline{U(L)}^{*}$, as in Proposition 6.2. The automorphism $\beta: U(K) \rightarrow U(K)$ is given as follows:

$$
\beta(b)=\left\{\begin{array}{l}
b+\operatorname{tr}(\overline{a d}(b)) \text { if } b \in K_{1}, \text { for } \overline{a d}(b) \in g l(L / K) \\
\left\langle g \mid g_{1} \cdots g_{n}\right\rangle b \text { if } b \in K_{g}, \text { for } g \neq 1,
\end{array}\right.
$$

where $\left\{x_{i}+K\right\}, i=1, \cdots, n, x_{i} \in L_{g_{i}}$, form a basis for $L$ over $K$. 
Proof. The first part of the corollary follows from Proposition 6.2 and our main theorem, 3.1, since $U(K) \star k G \subset U(L) \star k G$ is a $(B, A, H, A)$ faithfully-flat bi-Galois extension, with $H=k G, A=U(L) \star k G, B=U(K) \star k G, R=U(L)$, and $S=U(K)$. Thus $U(K) \subset U(L)$ is a $\beta$-Frobenius extension, with $\beta(b)=\sum \chi\left(b_{-1}\right) b_{0}$, where $\chi$ is described as in 6.2. The Frobenius homomorphism is $f=f_{\mathbf{1}}$, the integral in $\overline{U(L)}^{*}$, since $\bar{A} \cong \bar{R}$ by Lemma 5.5. Note also that we may use $\triangle_{U(L)}(r)$ in $f$ rather that $\triangle_{A}(r)$ by Theorem 5.6. Thus we need only check what $\beta$ does to elements of $K$.

For any $b \in K, \triangle_{R} b=b \otimes 1+1 \otimes b$. Passing to $B=U(K) \star H$, we have $\triangle_{A} b=\sum\left(b_{1} \star\left(b_{2}\right)_{-1}\right) \otimes\left(\left(b_{2}\right)_{0} \star 1\right)$. The $k G$-comodule structure on $K$ gives $b \mapsto g \otimes b$ if $b \in K_{g}$, and thus $\triangle_{A} b=b \otimes 1+g \otimes b$. Thus:

(a) if $b \in K_{1}, \beta(b)=\chi(b) \cdot 1+\chi(1) b=b+\chi(b) \cdot 1=b+\operatorname{tr}(\overline{a d}(b)) \cdot 1$, and

(b) if $b \in K_{g}$, for $g \neq 1$, then

$$
\beta(b)=\chi(b) \cdot 1+\chi(g) \cdot b=0+\left\langle g \mid g_{1} \cdots g_{n}\right\rangle b .
$$

Thus $\beta$ is as claimed.

6.4. Remark. (1) When $K \subset L$ are Lie superalgebras, we recover Bell and Farnsteiner's result $[\mathrm{BF}]$. For then $G=\mathbb{Z}_{2}=\{\overline{0}, \overline{1}\}$, and we see that our $\chi$ is exactly their $\lambda$ on $K$ itself. For the automorphism $\beta$, note that when $g=\overline{1},\left\langle g \mid g_{1} \cdots g_{n}\right\rangle=$ $\left\langle\overline{1} \mid \overline{1}^{n}\right\rangle=(-1)^{n}$, where $n=\operatorname{dim}(L / K)$, and thus $\beta(b)=(-1)^{n} b$ if $b \in K_{g}$. When $g=\overline{0}, \beta(b)=b+\chi(b) 1$ for $b \in K_{g}$, as before. This agrees with the automorphism $\alpha$ in $[\mathrm{BF}]$.

(2) If $K$ is a Lie ideal of $L$, that is $[K, L] \subset K$, then one might expect as in Example 1.11 that the extension $U(K) \subset U(L)$ is actually Frobenius, and not just $\beta$-Frobenius (for when $K$ and $L$ are ordinary Lie algebras, $K$ a Lie ideal of $L$ implies that $U(L)$ is a normal Hopf subalgebra of $U(L))$. However, this is not the case. It is true that $\overline{a d}(b)=0$, for $b \in K_{1}$, and thus $\beta(b)=b$, as in Corollary 6.3. But if $b \in K_{g}$, for $g \neq 1$, then $\beta(b)=\left\langle g \mid g_{1} \cdots g_{n}\right\rangle b$ as before; thus $\beta(b)=b$ only if $\left\langle g \mid g_{1} \cdots g_{n}\right\rangle=1$. For the case of superalgebras, this happens $\Leftrightarrow n$ is even.

The difficulty here is that $U(K) \star k G$ is not, in general, normal in $U(L) \star k G$, for $U(K)$ is not a Hopf subalgebra of $U(L) \star k G$ under the new comultiplication.

\section{NOTE ADDED IN PROOF}

M. Takeuchi has pointed out to us a more conceptual approach to Theorem 3.1. He defines a $\beta$-H-Frobenius extension to be an extension $B \subset A$ of right $H$ comodule algebras, which is $\beta$-Frobenius with Frobenius map $f$, such that $\beta$ and $f$ are $H$-colinear. For example $B \subset A$ in our 3.1 is $\beta$-H-Frobenius, and $U \subset W$ in our 1.14 is $\beta$-W-Frobenius. In the other direction he shows that if $U \subset W$ is a right coideal subalgebra of the Hopf algebra $W$ and if the extension is $\beta$-W-Frobenius, then it is an extension of right integral type.

Takeuchi proves the following: let $B \subset A$ be $\beta$-H-Frobenius, with $R=A^{\text {co } H}$ and $S=B^{c o H}$, and assume the following functor is a category equivalence:

$$
\begin{aligned}
\mathcal{M}_{S} & \rightarrow \mathcal{M}_{B}^{H} \\
M & \mapsto M \otimes_{S} B .
\end{aligned}
$$

Then $S \subset R$ is a $\beta$-Frobenius extension. 
Theorem 3.1 is a consequence of this result. Takeuchi's approach has been used by Y. Doi in "A note on Frobenius extensions in Hopf algebras", to appear in Communications in Algebra.

\section{REFERENCES}

[BMPZ] Yu A. Bahturin, A. A. Mikhalev, V. M. Petrogradsky, and M. V. Zaicev, Infinite Dimensional Lie Superalgebras, DeGruyter, Berlin, 1992. MR 94b:17001

[BF] A. D. Bell and R. Farnsteiner, On the theory of Frobenius extensions and its application to Lie superalgebras, Trans Amer. Math. Soc. 335 (1993), 407-424. MR 93c:17049

[Be] J. Bergen, Semisimplicity of restricted enveloping algebras of Lie superalgebras, Pacific J. Math 162 (1994), 1-11. MR 94k:17016

[CMZ] S. Caenepeel, G. Militaru, and S. Zhu, Doi-Hopf modules, Yetter-Drinfeld modules, and Frobenius-type properties, Trans. Amer. Math. Soc. 349 (1997), 4311-4342.

[Dr 86] V. G. Drinfeld, Quantum groups, Proc. ICM, Berkeley, 1986, vol. 1, 789-820. MR 89f: 17017

[F] R. Farnsteiner, On Frobenius extensions defined by Hopf algebras, J. Algebra 166 (1994), 130-141. MR 95e:16034

[FM] D. Fischman and S. Montgomery, A Schur double centralizer theorem for cotriangular Hopf algebras and generalized Lie algebras, J. Algebra 168 (1994), 594-614. MR 95h: 16051

[K] F. Kasch, Projektive Frobenius-Erweiterungen, Sitzungsber. Heidelberger. Akad. Wiss. Math.-Natur. Kl., 1960-61, 89-109. MR 24:A1932

[Ko] M. Koppinen, Coideal subalgebras in Hopf algebras: freeness, integrals, smash products, Comm. in Algebra 21 (1993), 427-444. MR 93j:16029

$[\mathrm{KT}] \quad$ H. F. Kreimer and M. Takeuchi, Hopf algebras and Galois extensions of an algebra, Indiana Univ. Math. J. 30 (1981), 675-692. MR 83h:16015

[LS] R. Larson and M. Sweedler, An associative orthogonal bilinear form for Hopf algebras, Amer. J. Math. 91 (1969), 75-93. MR 39:1523

[Mj] S. Majid, Doubles of quasitriangular Hopf algebras, Comm. in Algebra 19 (1991), 30613073. MR 92k:16052

[MD] A. Masuoka and Y. Doi, Generalization of cleft comodule algebra, Comm. in Algebra 20 (1992), 3703-3721. MR 93j:16030

[M] S. Montgomery, Hopf algebras and Their Actions on Rings, CBMS Lectures vol. 82, AMS, Providence, RI, 1993. MR 94i: 16019

[NT 60] T. Nakayama and T. Tsuzuku, On Frobenius extensions I, Nagoya Math. J. 17 (1960), 89-110. MR 23:A1687

[NT 61] T. Nakayama and T. Tsuzuku, On Frobenius extensions II, Nagoya Math. J. 19 (1961), 127-148. MR 25:3959

[NZ] W. D. Nichols and M. B. Zoeller, A Hopf algebra freeness theorem, Amer. J. Math 111 (1989), 381-385. MR 90c:16008

[OSch] U. Oberst and H.-J. Schneider, Über Untergruppen endlicher algebraischer Gruppen, Manuscripta Math. 8 (1973), 217-241. MR 50:339

[R 76] D. Radford, The order of the antipode of a finite-dimensional Hopf algebra is finite, Amer. J. Math. 98 (1976), 333-355. MR 53:10852

[R 85] D. Radford, The structure of Hopf algebras with a projection, J. Algebra 92 (1985), 322-347. MR 86k:16004

[R 93] D. Radford, Minimal quasi-triangular Hopf algebras, J. Algebra 157(1993), 285-315. MR 94c: 16052

[Sb] P. Schauenburg, Hopf bi-Galois extensions, Comm. in Algebra 24 (1996), 3797-3825. CMP 97:01

[Sche] M. Scheunert, Generalized Lie algebras, J. Math. Phys. 20 (1979), 712-720. MR 80f: 17007

[Sch 90] H.-J. Schneider, Principal homogeneous spaces for arbitrary Hopf algebras, Israel J. Math 72 (1990), 167-195. MR 92a:16047

[Sch 92] H.-J. Schneider, Normal basis and transitivity of crossed products for Hopf algebras, J. Algebra 152 (1992), 289-312. MR 93j:16032

[Sw] M. Sweedler, Hopf algebras, Benjamin, New York (1969). MR 40:5705 
[Tf] E.J. Taft, The order of the antipode of a finite-dimensional Hopf algebra, Proc. Nat. Acad. Sci. USA 68 (1971), 2631-2633. MR 44:4075

[T 72] M. Takeuchi, A correspondence between Hopf ideals and sub-Hopf algebras, Manuscripta Math 7 (1972), 251-270. MR 48:328

[T 79] M. Takeuchi, Relative Hopf modules - equivalences and freeness criteria, J. Algebra 60 (1979), 452-471. MR 82m:16006

[Wa] W. C. Waterhouse, Introduction to Affine Group Schemes, Springer-Verlag, Berlin, 1979. MR 82e: 14003

[We] A. Weil, L'intégration dans les groupes topologiques et ses applications, Hermann, Paris, 1938. MR 3:198b

[Y] D. N. Yetter, Quantum groups and representations of monoidal categories, Math. Proc. Cambridge Phil. Soc. 108 (1990), 261-290. MR 91k:16028

Department of Mathematics, California State University, San Bernardino, CaliforNIA 92407

E-mail address: fischman@math.csusb.edu

Department of Mathematics, University of Southern California, Los Angeles, CalIFORNIA 90089-1113

E-mail address: smontgom@math.usc.edu

Mathematisches Institut, Universität München, Theresienstrasse 39, D-80333 MuNICH, GERMAnY

E-mail address: hanssch@rz.mathematik.uni-muenchen.de 